\title{
CRYSTALLOGRAPHIC TOPOLOGY 2: OVERVIEW AND WORK IN PROGRESS
}

\author{
CARROLL K. JOHNSON \\ Oak Ridge National Laboratory, \\ Chemical and Analytical Sciences Division, \\ PO Box 2008, \\ Oak Ridge, \\ TN 37831-6197, USA \\ E-mail: ckj@ornl.gov
}

\begin{abstract}
This overview describes an application of contemporary geometric topology and stochastic process concepts to structural crystallography. In this application, crystallographic groups become orbifolds, crystal structures become Morse functions on orbifolds, and vibrating atoms in a crystal become vector valued Gaussian measures with the Radon-Nikodym property. Intended crystallographic benefits include new methods for visualization of space groups and crystal structures, analysis of the thermal motion patterns seen in ORTEP drawings, and a classification scheme for crystal structures based on their Heegaard splitting properties.
\end{abstract}

\section{Introduction}

Geometric topology and structural crystallography concepts are combined to define a research area we call Structural Crystallographic Topology, or just Crystallographic Topology. The first paper in the series[29] describes basic crystallography concepts (crystallographic groups, lattice complexes, and crystal structures) and their replacement topology concepts (orbifolds, topological lattice complexes, and Morse functions). To make the present paper self contained we discuss those topics again, but from a different perspective, so there is little direct repetition.

The new additions are: (1) the loosely defined Morse function used previously is replaced by a stochastic Morse function, based on the Radon-Nikodym property, which provides crystallographic thermal motion analysis capability as well as topological partitioning of global thermal motion density; (2) the topological lattice 


\section{DISCLAIMER}

Portions of this document may be illegible in electronic image products. Images are produced from the best available original document. 
complexes are extended to include the invariant, univariant, and divariant noncharacteristic (pseudo-symmetry site) orbits thus, providing better algebraic and pictorial characterization of crystallographic 3-orbifolds; and (3) a method for topological characterization and classification of crystal structures is introduced which uses Heegaard splitting of a Morse function (crystal structure) on a 3-orbifold (space group) into two handlebody 3-orbifolds separated by a Heegaard surface 2-orbifold.

For a known crystal structure, experimentally derived atomic thermal motion Gaussian density functions can be used to find all peak, pass, pale, and pit critical points and their stochastic separatrices, by using the Radon-Nikodym principle for pairs of neighboring atoms and stochastic negotiation for larger chemical clusters. This provides a critical net graph of the stochastic Morse function for global thermal motion density. We then add geometric representations for the rotation axes, inversion centers, and mirrors of the crystal's space group, and calculate all intersections (within a fundamental domain of the unit cell) with the stochastic Morse function Heegaard surface, which is a constant density surface partitioning (passes + peaks) and (pales + pits) into two disjoint sets.

The fundamental domain is topologically cut out and wrapped up to superimpose all symmetry equivalent boundary points, thus producing a Euclidean 3orbifold closed space representation of the crystal structure, space group, and Heegaard surface. The Heegaard surface provides Heegaard splitting[51] of the space group 3-orbifold and critical net into a pair of handlebody 3-orbifolds[65] with the shared 2-orbifold Heegaard surface (usually hyperbolic) between them.

To derive new (hypothetical) crystal structures, or classify those in existing data bases, one can transmute the Heegaard surface and critical net using various techniques of 3-manifold topology.[28,51] The transmutation procedure requires topological transformations. Our plan is to characterize these transformations by determining the topological differences between pairs of related known crystal structures, and we mention an existing topology procedures we plan to use for this, which involves Surf theory discriminants. $[49,50,39]$

The stochastic Morse function also provides a framework for stochastic thermal motion analysis[31] of ORTEP thermal ellipsoid patterns.[32] A full description is forthcoming for this vector-valued Gaussian measure approach, which is based on the Radon-Nikodym principle of absolute continuity between Gaussian measures.

\subsection{Background}

By topology we mean distortion invariant properties of spaces and objects rather than their topographic description. Our topological approach to structural crystallography strips away all metric detail. Instead we describe crystallographic geometry questions in terms of connectivity in a Morse theory sense, such as/ what is the smallest number of minimum negative gradient downhill and minimum positive gradient uphill path segments required to get from peak (atom) $A$ to peak $B$ in a thermal motion density map and how many equivalent paths exist?

The distortion invariance allows us to take a crystallographic unit cell from the infinite crystal and adjoin the three sets of matching faces to form a 3-torus (embedded into Euclidean 4-space) producing a closed topological space with more manageable mathematical properties than our original infinite Euclidean space. However, a unit cell will contain from 1 to 192 equivalent subunit polyhedra (i.e., fundamental domains or asymmetric units) depending on the space group of that crystal; thus it is more advantageous to "orbi-fold" the fundamental domain polyhedron by matching up all equivalent pairs of surface regions. This produces a Euclidean 3- 
orbifold, $\{61,12,13,29]$ which is the quotient space $E^{3} / G$ with $E^{3}$ Euclidean 3-space and $G$ one of the 230 space groups; thus we end up with the remnants of the space group (and crystal structure), modulo all symmetry, in the form of a closed space locally made up of differentiable (Hausdorff) submanifolds.

The orbi-folding eliminates the symmetry related repetition and puts all space groups on a more equal footing. Thus, the 3 -orbifold from a cubic space group has roughly the same complexity as that from a monoclinic example. However, we now find ourselves deep into the convoluted machinery of topology. The adjective deep is meaningful because very little theory in topology is near the surface. The 230 (or 219 affine space group orbifolds if handedness is ignored) Euclidean 3-orbifolds have an amazing variety of underlying topological spaces and we must learn to manipulate spaces such as $D^{3}$ (3-ball), $S^{3}$ (3-sphere), $R P^{3}$ (real projective 3-space), $R P^{2}$ suspensions, $S^{2} \times S^{1}$, lens spaces, solid Klein bottle, and others.

There are two basic methodologies used in topology. The first is combinatorial PL-topology (piece-wise linear), and the second is smooth manifold topology. The first uses subdivision such as triangulation, analysis of the subcomponents, and a tiling of the subcomponent results. PL-topology has many very powerful algorithms while smooth manifold topology has fewer methods available. However, our current plan is to continue our crystallographic topology studies in the smooth manifold domain since, in our view, this allows us to be compatible with a wider range of crystallographic theory and applications. Thus, in our present study we use Morse theory, Gaussian measures, Radon-Nikodym density, and Cerf theory rather than a possible alternative based on triangulation, tiling, Voronoi diagrams, and normal surface analysis.

There are also triangulation/tiling methods available for crystal structure prediction and classification which do not use either Morse theory or Euclidean 3orbifolds. For example, the D-symbol approach, described by Dress, Huson and Molnar,[10] uses the graphs of the singular sets for orbifolds separated from their underlying topological spaces. Friedrichs and Huson[19] find that there are 195 different "orbifold graphs" present in the 219 affine space groups and that abelian invariants can be used to differentiate space groups within the conflict sets.

\subsection{Organization Outline}

The crystallographic thermal motion analysis problem is described in section 2 , and an approach to this problem based on vector-valued Gaussian measures with the Radon-Nikodym property described in section 3. Radon-Nikodym density provides a partitioning between pairs of Gaussian measures suitable for defining the Morse function and critical net of section 4. Crystallographic groups are discussed in section 5, and crystallographic lattice complexes in section 6 . Underlying topological spaces, spherical 2-orbifolds, and Euclidean 3-orbifolds are discussed in section 7. Heegaard splitting of Euclidean 3-orbifolds and critical nets on 3-orbifolds is described in section 8, and example crystallographic Heegaard surfaces in the form of quadrilateral Haken normal surfaces shown in section 9. A tabulation of crystallographic Heegaard surfaces in the appendix is described in section 10, and suggestions for further research given in section 11.

\section{Crystallographic Methods and Published Results}

A high precision crystal structure analysis of a small (1-50 atoms in fundamental domain) to medium (50-300 atoms) crystal structure routinely includes refinement 


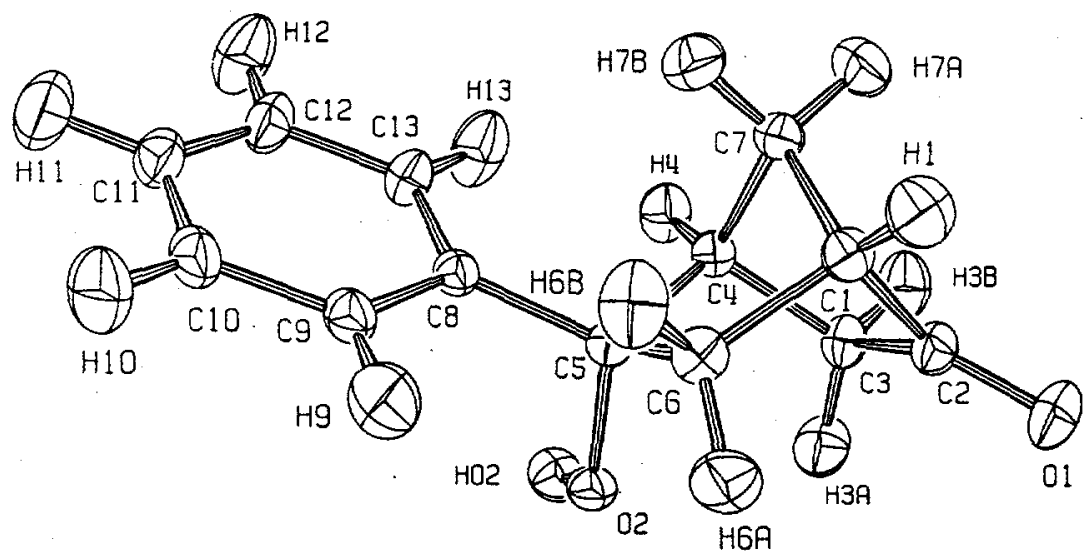

Figure 1: Phenylhydroxynorbornanone (0.5 Probability Ellipsoids)

of six anisotropic temperature factor parameters, in addition to the three mean positional parameters, per atom. With proper scaling, these thermal motion parameters for an atom form the symmetric $3 \times 3$ cumulant matrix for the characteristic equation of a trivariant normal probability density function, which is herein referred to as a Gaussian measure[60] since we sometimes use it in a stochastic process context in this paper.

Experimental determination of the dynamic correlation between the motions of various atoms and the mathematical modeling required is usually rather expensive and time consuming; thus, crystallographers usually use much simpler procedures for interpretation of their thermal motion ellipsoid patterns without resorting to spectroscopic measurements and normal mode analysis, or neutron scattering measurements and lattice dynamics interpretation.

\subsection{Thermal Ellipsoid Patterns}

Many crystal structure papers include a thermal ellipsoid drawing (often made by our ORTEP[14] program) of selected atom clusters such as a molecule, if present, as shown in Figure 1. The ellipsoids represent a constant density surface for the atomic Gaussian measure and enclose a stated fraction of the total probability density, often 0.5 . The global pattern of thermal ellipsoids in the drawing provides a visual summary of the time and lattice averaged thermal displacements of the atoms. Prominent modes of molecular or lattice vibrations, such as the wag of a carbonyl group, are often readily apparent from the figure. Some readers use the reasonableness of the pictorial ellipsoid pattern to judge overall reliability for a published crystal structure before studying the numerical results.

\subsection{Mechanistic Models for Thermal Motion}

There are mechanistic thermal motion analysis models available such as the Schomaker and Trueblood rigid body motion model[58] and its various extensions such as our 
segmented rigid body model, $[30]$ but these are not used routinely because the diversity of major large amplitude thermal displacement modes in crystals makes analysis of rigid body calculation results difficult. However, these simple mechanistic models can sometimes provide interesting information. ${ }^{a}$

\subsection{Crystallographic Density Maps}

Topological studies of electron density maps experimentally derived using $\mathrm{x}$-ray diffraction (which sees electrons) are now an important tool in crystallographic charge density studies. Quantum chemistry effects such as lone pair electron densities between covalently bonded atoms are often readily observable in such maps, particularly for experimental results at very low temperatures where thermal motion is minimal. Studies using neutrons rather than $x$-rays produce nuclear density rather than electron density maps since neutrons see nuclei rather than electrons; consequently, joint $x$-ray and neutron studies are advantageous for a cleaner separation of the thermal motion effects from the quantum wavefunction effects. Figure 1 is from our unpublished neutron diffraction study, which insures reliable thermal ellipsoids for even the light hydrogen atoms that are troublesome in $\mathrm{x}$-ray studies.

One can also work with calculated thermal motion density maps by summing all the atomic Gaussian density functions, then numerically finding the critical points[22] and their topological separatrices in the summed thermal motion density function. Such a thermal motion map does not have the noise (experimental and quantum) present in the experimentally derived electron density maps.

There is a significant difference between observed (or calculated) map critical point[22] (and thermal motion) analysis and the stochastic critical point (and thermal motion) approach described next. The stochastic approach does not sum the individual Gaussian density functions but instead works with multiple pairwise differences to determine stochastic, rather than topological critical points and separatrices. The critical point analysis features of the results from these two approaches are similar but the analysis details are quite different. The stochastic approach also provides some interatomic motion correlation results without superimposing mechanistic assumptions.

\section{Radon-Nikodym Density}

While researching thermal ellipsoid pattern interpretation problems, we found some interesting stochastic process results involving the Radon-Nikodym derivative of one measure with respect to a second measure, which for Gaussian measures[60] is a classic concept in mathematical analysis[52] and probability theory.[23,46] When used in a probability setting, the derivative is called the Radon-Nikodym density. Much has been written on the subject of when can this be done, particularly the

\footnotetext{
a The rigid-body mean-square-displacement model has 21 parameters in a $6 \times 6$ symmetric "TLS" matrix with $3 \times 3$ submatrices for translation $T$, libration $L$, and screw coupling $S$. The parameters are adjusted by least-squares refinement to the experimental pattern of thermal ellipsoids. The segmented rigid-body has a series of rigid groups arranged as branches on a tree with upper branches "riding" on lower branches. Flexible joints between segments are constrained by adjusting only selected parameters of the associated TLS matrix. For the molecule in Figure 1, two segments were used with the phenyl group riding on the norbornanone cage. After subtracting out meansquare displacements estimated for the hydrogen atoms' internal molecular motion, a satisfactory fit to the experimental thermal ellipsoids was obtained. The rather unusual pattern of ellipsoids on the phenyl group in Figure $I$ is due to screw components in the phenyl TLS, which may physically arise from a buckling distortion within the cage (i.e., as the body hiccups, the molecule tosses its head and twists its neck).
} 
books by Diestel and Uhl[11] and Bourgin.[4] The main criterion is that there be "absolute continuity of two Gaussian measures defined on the same Hilbert space," which for crystal structure thermal motion is satisfied by the fact that we have pairs of Gaussian measures interacting in the same physical Euclidean 3-space. We call the use of this equation for coupled thermal motion the stochastic thermal motion approximation.

\subsection{Two Interacting Gaussian Measures}

Derivation of the following equations is given in Gihman and Skorohod[23, pp 486]. Also see Richter[46, pp 44] for a likelihood function multi-Gaussian measure extension.

The characteristic equations for Radon-Nikodym density, in orthogonal covariant coordinates of crystallographic reciprocal space,

$$
\Phi_{k}(z)=\exp \left(i a_{k}^{t} z-\frac{1}{2}\left(z^{t} B_{k} z\right)\right), k=1,2
$$

define two adjacent atoms with mean vectors $a_{1}, a_{2}$ and temperature factor matrices $B_{1}, B_{2}$. A modern description of the various crystallographic structure factor equations which can be used to derive these quantities is given by Coppens.[9] The (direct space) Gaussian densities in contravariant components are:

$$
\mu_{k}(x)=\frac{\left|B_{k}^{-1}\right|}{(2 \pi)^{3 / 2}} \exp \left(-\left(x-a_{k}\right)^{t} B_{k}^{-1}\left(x-a_{k}\right)\right), k=1,2
$$

Using $B_{2}^{-1 / 2}$ as a transformation matrix, we define

$$
\begin{gathered}
b=B_{2}^{-1 / 2}\left(a_{2}-a_{1}\right), \\
c(x)=B_{2}^{-1 / 2}\left(x-a_{1}\right), \\
D_{2,1}=B_{2}^{-1 / 2}\left(B_{1}-B_{2}\right) B_{2}^{-1 / 2},
\end{gathered}
$$

where $D_{2,1}$ is a Hilbert-Schmidt matrix (for atom 2 with respect to the reference atom 1) with 3 eigenvalues and eigenvectors denoted $\lambda_{k}$ and $e_{k}, \mathrm{k}=1,2,3$, respectively. The Radon-Nikodym density, $\omega_{2,1}(x)$, defined as the derivative of $\mu_{2}(x)$ with respect to $\mu_{1}(x)$ is given by the equation

$$
\frac{d \mu_{2}}{d \mu_{1}}(x)=\exp \left(-\frac{1}{2}\left(\sum_{n=1}^{3}\left(e_{k}^{t} c(x)\right)^{2} \frac{\lambda_{k}}{\left(1+\lambda_{k}\right)}-\ln \left(1+\lambda_{k}\right)\right)+c^{t}(x) b-\frac{b^{t} b}{2}\right) .
$$

When we set

$$
\frac{d \mu_{2}}{d \mu_{1}}(x)=\omega_{2,1}(x)=1=\frac{1}{\omega_{2,1}}(x)=\omega_{1,2}(x)=\frac{d \mu_{1}}{d \mu_{2}}(x),
$$


we obtain the equation for the topological separatrix surface separating the two Gaussian thermal motion measures $\omega_{2,1}(x)$ and $\omega_{1,2}(x)$. In addition, for any point $y$ on the separatrix,

$$
\mu_{1}(y)=\mu_{2}(y) \Longleftrightarrow \omega_{2,1}(y)=\omega_{1,2}(y)=1 .
$$

\subsection{Radon-Nikodym Gradient Fibers}

The gradient fibers in the $\omega$ (i.e. Radon-Nikodym) density space are of stochastic importance. At the $a_{1}$ site, $\omega_{2,1}(x)$ density is a minimum and increases along an $\omega_{2,1}(x)$ fiber which terminates at the separatrix with $\omega_{2,1}(x)=1$. At that point another fiber, $\omega_{1,2}(x)$, starts with a value of 1 and decreases in value to a minimum at the $a_{2}$ site. Gradient $\omega$ fibers radiate in all directions from the two Gaussian centroids, and for $t=1$ they all terminate at the separatrix. The solid elliptical cone angle of gradient fibers meeting the separatrix increases from an vanishingly small number to $2 \pi$, as $t$ increases from $t_{1}$ to 1 . Thus, Radon-Nikodym interaction between Gaussian measures is proportional to the separatrix area between them, or by Stokes rule, proportional to the perimeter of that area.

\subsection{Three Interacting Gaussian Measures}

The above two-Gaussian-measure results may be extended to three measures by the differentiation chain rule

$$
\frac{d \mu_{2}}{d \mu_{1}}=\frac{d \mu_{2}}{d \mu_{3}} \frac{d \mu_{3}}{d \mu_{1}}
$$

If the above equations are generalized to have an arbitrary origin and coordinate system and all three $\omega$ densities are simultaneously unity in that coordinate system, the three separatrices intersect. Thus, we obtain the surprisingly simple result

$$
\mu_{1}=\mu_{2}=\mu_{3} \Longleftrightarrow \omega_{2,1}=\omega_{2,3}=\omega_{3,1}=\omega_{1,2}=\omega_{3,2}=\omega_{1,3}=1 .
$$

This result, which can be generalized even further, has interesting crystallographic symmetry implications as described in section 4.2 .

Given three Gaussian measures in a triangular arrangement, we obtain three separatrix surfaces, one between each of the three measures. If the three Gaussians are related by a 3-fold axis, the separatrices will intersect on that 3-fold axis, and there are a number of less symmetrical possibilities, some analogous to the 2dimensional expanding circles discussed by Siersma.[56] However, we will not pursue that approach, since in 3-dimensions the tetrahedron is the simplest fully symmetric configuration, which we discuss below in the Morse Theory section.

\subsection{Vector-Valued Gaussian Measures}

In crystals the minimum configuration of interest will always be three or more neighbors around any reference ellipsoid; thus vector-valued measure theory is required $[11,4,40]$ We may consider a vector-valued probability measure $\mu=\left(\mu_{1}, \ldots, \mu_{s}\right)$, where all component measures are pairwise mutually absolutely continuous, or one where only the neighboring measures $\mu_{k}$ are pairwise absolutely continuous, while the more widely separated measures have $\omega=0$.[60] For thermal motion analysis we would use fairly large local "coherence zones", but for the present topology application we simply use local zones large enough to partition space into polyhedra 
around the atoms. These cage-like polyhedra are called dented bodies or dentable sets by Diestel and Uhl, [11] and Bourgin.[4] The term dentable carries a stochastic meaning: if a set of measures is not dentable, it does not have the Radon-Nikodym property. We refer the reader to the above references, and Ledoux and Talagrand[40] for details.

\section{Morse Functions and Critical Nets}

A critical point of a smooth function $f$ occurs at $x$ if and only if $(\partial f / \partial x)=0$ and $\left(\partial^{2} f / \partial^{2} x\right)=M$, with $M$ a $3 \times 3$ symmetric matrix with non-zero determinant $(|M| \neq 0)$. For convenience we name the critical points, "peak", "pass", "pale", or "pit" if the eigenvalues of $M$ have the sign sets $(-,-,-),(-,-,+),(-,+,+)$, or $(+,+,+)$, respectively. A Morse function is a differentiable function on a smooth 3-manifold which has no degenerate critical points (i.e., no zero eigenvalues). A Morse function is homotopy equivalent to a CW-complex (i.e., closure finite, weak topology, cell complex). We represent a crystallographic Morse functions as a critical net, which is a 1-dimensional (graph) representation of the CW-complex. The critical net gives all minimum gradient paths connecting peaks to passes to pales to pits. The maximum gradient paths between peaks and pits also could be included[21, see figure in final chapter] but we always omit them from illustrations.

\subsection{Probability Flow Scenarios}

Crystallographers might visualize the following model as simply a series of modified ORTEP drawings made at increasing probability levels. The modification is that the 4th-order intersection curve[57, pp 101-105]of two adjacent ellipsoids is calculated at high probability levels where overlap occurs, and is the only feature drawn. In addition, simultaneous intersection of three or more ellipsoids are calculated[1, Ch.7] and drawn. The scenarios may aid construction of an algorithm for computing critical point graphs, if an incremental separatrix growth approach is taken.

1. We can consider the Gaussian measure mean sites $a_{1}, a_{2}$ to be ellipsoidal wavefront sources producing expanding thermal ellipsoids over a time interval $t=0: 1$, with total enclosed density for each ellipsoid equal to $t$. Thus at $t=0.5$ the total enclosed density is 0.5 . At time $t_{1}$, the two ellipsoids touch at a single point $y_{1}$. In the time interval $t_{1}: 1$ the separatrix surface expands outward to infinity from $y_{1}$ to form a curved, but not closed, surface. If the two thermal ellipsoids are related by a mirror of symmetry, the separatrix surface will be planar.

2. Now assume we have four identical spherical Gaussian measures on the vertices $a_{1}, a_{2}, a_{3}, a_{4}$ of a regular tetrahedron. We again use expanding probability as a scenario generator:

(a) At $t=t_{0}$, spherical wavefronts start expanding in 3-dimensions about the four vertices of the tetrahedron (the 4 peaks).

(b) At $t=t_{1}$, spherical wavefronts from two neighboring vertices collide at the center of each of the six edges of the tetrahedron (the 6 passes).

(c) At $t=t_{1}+\epsilon$, six two-dimensional separatrix surfaces between pairs of spherical wavefronts start to develop (as circular separatrix-fronts) normal to each of the six edges of the tetrahedron with a pass as its center. 
(d) At $t=t_{2}$, three circular separatrix-fronts from three passes collide at the center of each of the four triangular faces of the tetrahedron (the 4 pales).

(e) At $t=t_{2}+\epsilon$, one-dimensional triseparatrix lines between three spherical wavefronts start to develop (as vectorial trisepratrix-fronts) perpendicular to each face of the tetrahedron, and extending from a pale.

(f) At $t=t_{3}$, the four spherical wavefronts, six circular sepratrix-fronts, and three vectorial triseparatrix-front lines all collide simultaneously at the center of the tetrahedron (a pit) $)^{b}$.

\subsection{Geometric aspects of Radon-Nikodym Property}

The above tetrahedron can be distorted somewhat and the spherical distributions replaced by anisotropic Gaussians without changing the topology of 4 peaks, 6 passes, 4 pales and 2 pits.

1. The only exact change possible is an ellipsoidal expansion of the vertices about the center of the tetrahedron, and that distortion ellipsoid shape then used as wavefront generators at the four vertices.

2. More complex distortions of the tetrahedron will require four different ellipsoid wavefront generators. In addition, the triseparatrix lines may no longer be single, and the critical levels $\left(t_{0}, t_{1}, t_{2}, t_{3}\right)$ may form bands of discrete levels. It then becomes necessary to "stochastically negotiate" a triseparatrix line or alter the Morse function. This negotiation is a key factor in the stochastic thermal motion analysis to be described in a later publication.

3. All atoms related by symmetry have their positions and thermal ellipsoids related by that symmetry, and symmetry equivalent atoms will have separatrices on all the special position Wyckoff point, line, and plane sites of their space group. The simplest applications of the Radon-Nikodym property will be for simple high symmetry crystal structures, particularly those with octahedral, tetrahedral, and dihedral crystallographic point group operators. A related tetrahedron example was discussed in a previous section.

4. One can also partition each Gaussian measure into two components:[23] the first with Radon-Nikodym coherence and the second without that coherence. This assumption is equivalent to the Busing and Levy [3] riding model which we use in our segmented rigid body model.[30]

5. The fact that we can also consider "normal modes", with independent coherence, leads to considerable flexibility in thermal motion analysis through use of multiple vector-valued Gaussian measures as will be discussed is a separate paper.

\footnotetext{
${ }^{b}$ Actually there are two pits - the second is on the point at infinity for the Euclidean representation of the 3-sphere $S^{3}$, since we can really only count critical points in a closed manifold such as $S^{3}$. A second possibility is to put mirrors on all faces of the tetrahedron forming a 3-disk (3-ball), $D^{3}$, which would reduce the count to 2 peaks, 3 passes, 2 pales, and 1 pit, since the count on mirrors is divided by two. A third critical point bookkeeping possibility is to consider only the surface polyhedron ( $S^{2}$ topology) and use the Euler relation vertices - edges + faces $=2$. The 3torus and the 3 -sphere each have the Euler-Poincare relationship peaks - passes + pales - pits $=0$ (i.e., $\chi=0$ ), while the 2-sphere has $\chi=2$. $\chi$ is called the Euler characteristic.
} 

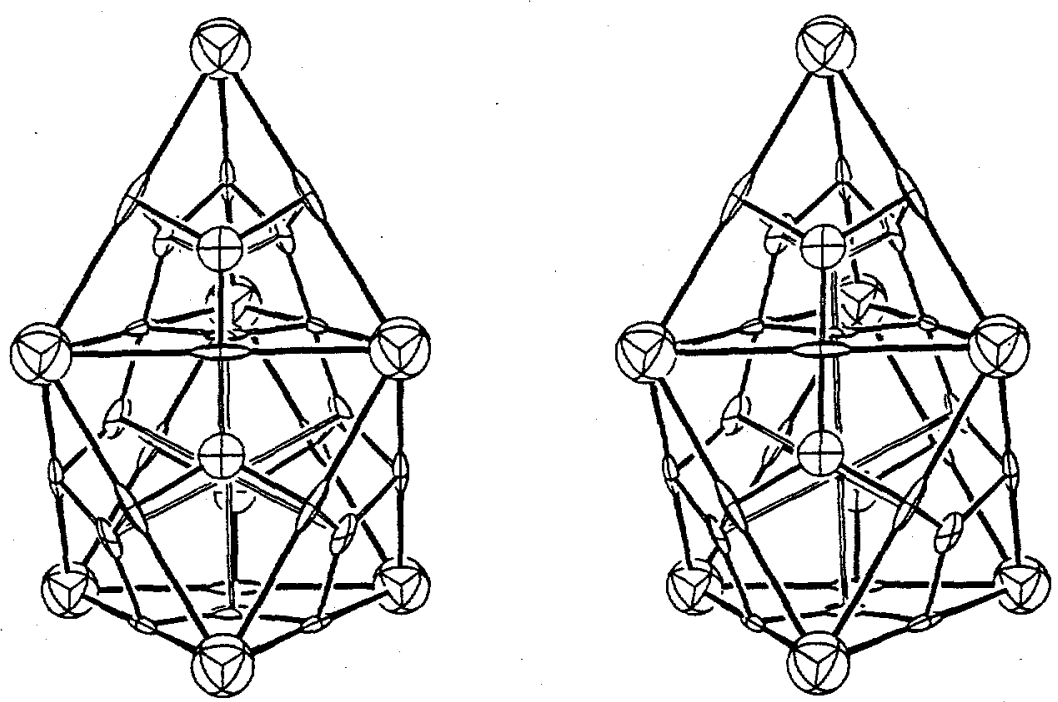

Figure 2: Stereo Drawing of Face Centered Cubic Critical Net, in $F m \overline{3} m$

\subsection{Critical Net Graphs}

The critical net graph has critical points as graph vertices and topological lines between the successive types of critical points as graph lines. Figure 2 is a stereoscopic pair of drawing illustrating the critical net for the face centered cubic (FCC) type of crystal structure (e.g., copper) which contains both tetrahedral and octahedral polyhedra, with one of each shown in the drawing. In our critical net drawings, ellipsoids are used to distinguish the four types of critical points (rather than to represent thermal motion as in Figure 1). The large spheres, elongated ellipsoids, flattened ellipsoids, and small spheres represent peaks, passes, pales, and pits, respectively.

Figure 3 shows dilithium oxide, $\mathrm{Li}_{2} \mathrm{O}$, the dual of $\mathrm{FCC}$, where the peaks and pits (also passes and pales) are interchanged so there is one "chemical cage" polyhedron with two types of atoms. The largest sphere for oxygen and the next largest for
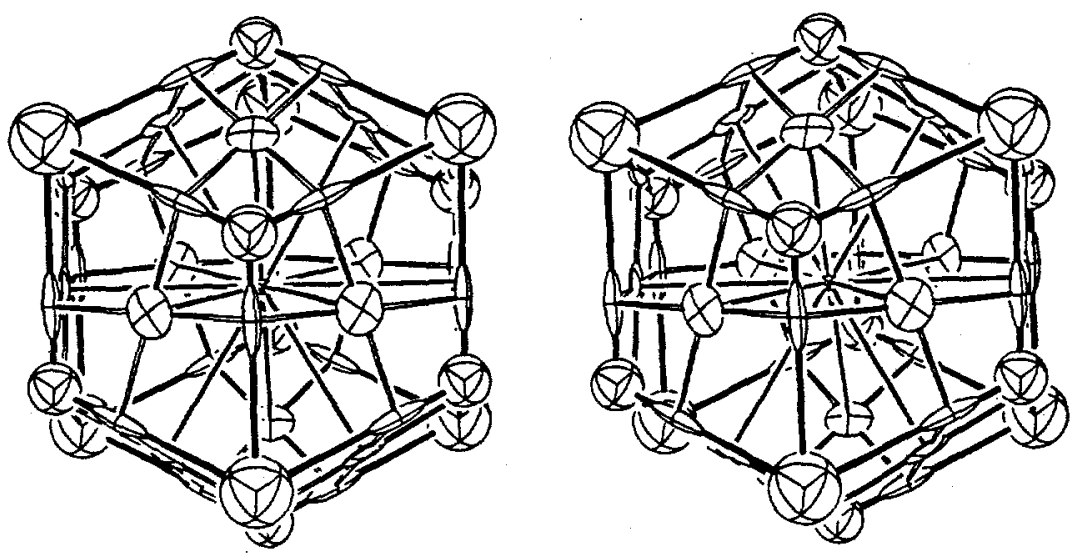

Figure 3: Stereo Drawing of DiLithium Oxide Critical Net, in $F m \overline{3} m$ ) 


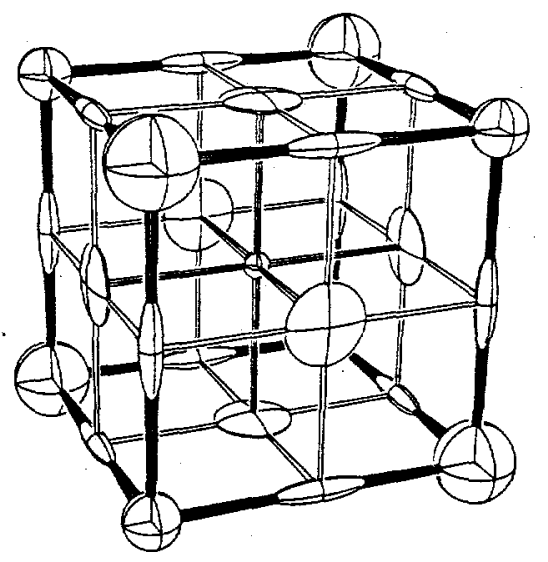

Figure 4: Sodium Chloride Critical Net

lithium. The pale of FCC is arbitrarily shown as coplanar within the tetrahedron and octahedron faces of Figure 2, but is required by symmetry only to be on the 3 -fold symmetry axis between the two pits. If the pale is centered between pits in Figure 2, the pass is centered between peaks for its dual in Figure 3. The centered position is called a non-characteristic or pseudo-symmetry site (see Section 6.2).

Figure 4 show the critical net for sodium chloride (space group $F m \overline{3} m$ ), but if both peaks are identical it becomes the simple cubic structure structure $(P m \overline{3} m)$. For simple structures such as the above, all or most of the critical points are on special positions of the crystal's space group. For these fixed point structures the critical net can be determined through the rules stated in the next subsection.

For a more complex crystal structure, such as that shown in Figure 1, which has no atoms in special positions, the Radon-Nikodym calculations described above can be used with averaging for the cases where minor splitting of critical points or separatrices occur, assuming the crystal structure parameters are available.

\subsection{Morse Theory}

Morse theory[21,54,55] explores questions such as: What does a manifold know about the Morse function on that manifold, and vice-versa? For a crystal structure Morse function, let the manifold be a 3-torus (unit cell) with given space group symmetry. If the space group has singularities, they must be compatible with the critical net of the Morse function. We find the following Euclidean 3-space characterizations useful.

1. Morse Inequalities: Let $n_{0}, n_{1}, n_{2}, n_{3}$ be the number of peak, pass, pale, and pit critical points, respectively, in the Morse function on the 3-torus. The Morse inequalities for the unit cell contents are: $n_{0}-n_{1}+n_{2} \geq 1, n_{3}-n_{2}+n_{1} \geq$ $1, n_{0}-n_{1} \geq 2, n_{3}-n_{2} \geq 2, n_{0} \geq 1, n_{3} \geq 1$, and the Morse equality is

$$
n_{0}-n_{1}+n_{2}-n_{3}=0 \text {. }
$$

The equation and inequalities are helpful constraints on verifying that we have a true Morse function and have found all the critical points. The equality also provides a calculation of the Euler-Poincare characteristic, which is the expected $\chi=0$ of Euclidean 3-space, but here we are using Morse function critical points rather then the usual Betti numbers as coefficients. 
2. Fixed points: If the space group has fixed point singularity sites, then one and only one critical point of the Morse function must reside on each of these sites. Any remaining critical points of the Morse function will be positioned on lower symmetry sites.

3. Critical Net Arcs: The arcs of the Morse function critical net represent gradient lines; thus they cannot pass through a fixed point symmetry site, rotation axis, or mirror of the space group since that would generate an additional Morse function critical point at the point of intersection.

4. Wagon Wheels: We observe that crystallographic critical nets have wagon wheels of pales around peak - pass - peak linear axles and wagon wheels of passes around pit - pale - pit linear axles with minor distortions allowed. This may be related to the transversality theorems of Morse theory. The wagon wheels observed to date have $3,4,5$, and 6 spokes, with the 5-spoke example being a hypothetical Morse function which fits onto the B-type [29] cubic space groups.

5. Wagon Wheel Axles: A useful Morse function constraint based on the wagon wheel property is that there must be one and only one wagon wheel axle at each peak and pass. We can express this in terms of the "Q rule": $Q_{1}$ (resp., $Q_{2}$ ) = (order of Wyckoff multiplicity on pass-to-peak arc (resp., pale to pit arc)) / (order of the Wyckoff multiplicity on pass (resp., pale)) $=2$, if and only if the pass (resp., pale) is not degenerate. Instead of using Wyckoff multiplicity ratios, one can use group order ratios which is the convention used in the mathematical literature. Keep in mind that the two ratios are reciprocal.

\section{Crystallographic Groups}

The crystallographic groups [24] describe discrete rigid motions. A $n$-dimensional space group has a normal free Abelian subgroup of rank $n$, which is maximal Abelian and has finite index.[26].

\subsection{The Unit Cell}

Each 3-dimensional space group has one of 14 different Bravais lattices as the Abelian subgroup, $L$, mentioned above. The quotient $G / L=T^{3}$, with $T^{3}$ a 3torus group, defined as the direct product of 3 copies of the torus group $T=R / Z$, with $R$ the additive group of real numbers and $Z$ the additive group of rational integers. The 3-torus concept may be interpreted in two different ways. First, it is considered as $T^{3}=$ I $\times$ I $\times$ I ( $\mathrm{I}=$ interval) with cyclic boundary conditions where at any time the size of each $I$ is arbitrarily set to include as many copies of the parallelepiped unit cell as we wish for the calculation or illustration at hand. Secondly, it may be considered a topological torus where the cell is distorted to bring each of the three opposite pairs of unit cell faces into physical contact $T^{3}=S^{1} \times S^{1} \times S^{1}$. The two models are homeomorphic. The first interpretation is the traditional one used in distortion-sensitive metric crystallography. However, we normally use the second interpretation in distortion-invariant crystallographic topology.

$T^{3}$ is the finite unit cell for the infinite space group $G$, and contain $m$ ( $m=1$ to 192) symmetry equivalent fundamental domain (asymmetric unit) polyhedra. $m$ is the general position Wyckoff multiplicity of the unit cell. There may also be special 
Wyckoff sites (points, lines, and planes) in the unit cell. Each will have Wyckoff multiplicity $m / q$ where $q$ is the order of the local symmetry group (on that site), which is one of the 32 point groups discussed below. Each of these special Wyckoff sites in the unit cell represents a characteristic orbit of the space group.

\subsection{Nomenclature}

A space group $G$ projected along one of its three distinctive non-parallel axes (choice depending on the crystal class: i.e., triclinic, monoclinic, orthorhombic, tetragonal, trigonal, hexagonal, or cubic) gives one of the 17 infinite plane groups $J$, sometimes called wallpaper groups. The three plane group projections, $J_{j}: j=1,2,3$, are not necessarily different. A symbol for the Bravais lattice, $L$, and a distinctive space group generator from each of the three projection axes, are adjoined to form the space group symbol.

Example - A space group's full symbol, $F \frac{4_{1}}{d} \overline{3} \frac{2}{m}$,(normally shortened to $F d \overline{3} m$ ) tells us that this is a cubic space group (orthogonal axis $a=b=c$ ) with (a) face centered unit cell (F); (b) $4_{1}$ screw axis along $a$, with diamond glide planes perpendicular to $a$; (c) $\overline{3}$ axis along the $a+b+c$ body diagonal; and (d) 2 -fold axis parallel to the $b+c$ face diagonal, and a mirror plane perpendicular to that axis.

\subsection{Classification}

Space groups, G[24], stripped of all translation components other than their Bravais lattices, project to the 73 arithmetic crystal classes[63], $A$, which modulo the Bravais lattices become the 32 geometric crystal classes (i.e., point groups: discrete cyclic (2, 3,4 , and 6 only) dihedral, tetrahedral, and octahedral members of the orthogonal group $O(3)), K$. The classification hierarchy may be represented by surjective (onto) mapping arrows in a commutative diagram,[26]

$$
G(230) \rightarrow G_{a}(219) \rightarrow A(73) \stackrel{\rightarrow K(32) \rightarrow}{\rightarrow L(14) \rightarrow} C(6),
$$

with $G_{a}$ the affine space groups (handedness ignored), and $C$ the coproduct of $K$ and $L$ over $A$. As an example,

$$
P 6_{5} 22 \rightarrow P 6_{1,5} 22 \rightarrow 622 P \underset{\rightarrow P 22 \rightarrow}{\rightarrow P \rightarrow} \text { Hexagonal. }
$$

There is also a group extension (short exact sequence) $[2,62]$

$$
0 \rightarrow L \rightarrow G_{s} \rightarrow K \rightarrow 1
$$

where $G_{s}$ is the symmorphic (no screw axes or glide planes) member of the arithmetic crystal class. Short exact sequences have interesting mathematical properties we will not explore here. However, it tells us that $K=G_{s} / L$. There appears to be no simple mathematical algorithm which generates $G$ from $K$ and $L$.

\section{Lattice Complexes}

Lattice complexes have been known to crystallographers for over 70 years, but have not been used much in recent years. However, we find them to be invaluable landmarks for the crystallographic orbifolds. 


\subsection{Characteristic Lattice Complexes}

1. Invariant Lattice Complex:[24,17,29] These sites are all the fixed point Wyckoff sites in a space group unit cell, and all lie on a superlattice within that unit cell, which for the cubic crystal family divides the cell into $8 \times 8 \times 8$ parts. Various patterns are formed on that superlattice, and each pattern is given a one letter name[17] which characterizes that pattern. Translation of the pattern, expressed in multiples of $1 / 4,1 / 4,1 / 4$ within the cell; is indicated by the number of postfix primes. Prefix symbols $\left({ }^{\prime},+,-\right.$, etc.) indicate other transformations, and a subscript 2 , such as $P_{2}$, indicates doubling of the lattice complex in all 3 directions within the cell.

2. Invariant Lattice Complex Nomenclature[17,24] and Algebra[17,29]: For the cubic family there are 16 invariant lattice complexes. Their nomenclature and algebraic relations follow: $D$ (diamond) $=F+F^{\prime}, D^{\prime \prime}=F+F^{\prime \prime} ; F$ (face centered $)=P+J, F_{2}^{\prime}=T+T^{\prime \prime} ; I$ (body centered $)=P+P^{\prime \prime}, I_{2}=D$ $+D^{\prime \prime}=P_{2}+P_{2}{ }^{\prime} ; J($ jack $), J^{*}=J+J^{\prime \prime} ; P$ (primitive); $P_{2}=F+F^{\prime \prime} ;$ $S$ (Schoenflies $\left.S_{4}\right), S^{*}=S+{ }^{\prime} S ; T$ (tetrahedral), ${ }^{+} V,-V$ (Schoenflies $\left.V\right), V^{*}$ $=+V+-V ; W$ ("non-intersecting rows"), $W^{*}=W+W^{\prime \prime}, W_{2}=V^{*}+S^{*}$; $+Y,-Y$ ("Y-shaped"), ${ }^{+} Y^{*}={ }^{+} Y+{ }^{+} Y^{\prime},-Y^{*}=-Y+{ }^{-} Y^{\prime}$, and $\mathrm{Y}^{* *}=$ $+Y^{*}+-Y^{* \prime \prime}$.

3. Characteristic Univariant, Divariant, and Trivariant Lattice Complexes: These are lines with an invariant member on each end, mirror planes containing a graph of univariant members, and the entire set of invariant, univariant, and divariant members, respectively.[17,37,24] In the cubic space groups, each trivariant lattice complex is unique to one space group, but this is not the case in other crystal families. A divariant, univariant, or invariant lattice complex can occur in several different space groups. ${ }^{c}$

\subsection{Non-Characteristic Lattice Complexes}

A characteristic invariant lattice complex of a space group may or may not appear as a characteristic lattice complexes in the subgroups (i.e., daughter space groups) of the parent space group. If it disappears but the neighboring lattice complexes it is connected to in a monovariant lattice complex do not disappear. it becomes a non-characteristic (pseudo-symmetry) invariant lattice complex, A more formal description follows.

The set of points generated from a point $X_{0}$ by the space group $G$ is called a crystallographic orbit $\Lambda\left(X_{0}\right)$. If $E$ is the symmetry (eigensymmetry) of this set of points and $E=G$, then $\Lambda\left(X_{0}\right)$ is a characteristic crystallographic orbit. However if $E$ is a supergroup of $G$, then $\Lambda\left(X_{0}\right)$ is a non-characteristic crystallographic orbit with respect to $G$. These are tabulated in "The Non-characteristic Orbits of the Space Groups" by Engel et. al.[15], and in papers by Koch[36], and by Fischer and Koch.[18] Non-characteristic orbits[15] are not exactly the same as limiting lattice complexes[38] discussed in the lattice complex literature but the main differences seem to disappear in the crystallographic topology application.

\footnotetext{
${ }^{c}$ There are 16 unique invariant cubic lattice complexes in the 35 affine cubic space groups and 20 more in other crystal classes for a total of 36 in the 219 affine space groups. There are also 44 monovariant, 16 divariant, and 35 trivariant lattice complexes in the cubics and 62 more monovariant, 89 more divariant, and 120 more trivariant lattice complexes in the remaining 184 affine space groups. Thus there are 402 lattice complexes in the 230 space groups.[17]
} 
1. Non-characteristic Invariant Lattice Complexes:[36,15] These are points of pseudo-symmetry which are often the preferred sites occupied by Morse function critical points after the characteristic invariant sites of a space group have been filled. They also provide additional landmarks for orbifold visualization and algebraic characterization.

2. Non-characteristic Univariant, Divariant, and Trivariant Lattice Complexes: The univariant and divariant non-characteristic lattice complexes (orbits) are included in our on-line Euclidean 3-orbifold atlas.[33]. They are useful for pseudo-symmetry crystallographic problems such as disorder, in addition to providing convenient orbifold landmarks.

\subsection{Wyckoff Sets}

The different Wyckoff positions of a space group $G$ may permute under an isomorphic mapping of $G$ onto itself (i.e., under an automorphism of $G$ ). A Wyckoff set is the collection of all Wyckoff sites that may be permuted by automorphisms of G.[24, Sect. 8.3.2].

When an orbifold has more than one characteristic lattice complex with the same topology lattice complex notation (defined below), they are members of the same Wyckoff set. This is true for invariant, univariant, and divariant characteristic lattice complexes.

\subsection{Topology Notation for Lattice Complexes}

The invariant lattice complex notation transfers directly to crystallographic topology. However, the primes referring to translations in space-group space are omitted since that information is implicit in the singular set graphs of orbifold drawings. We found the standard crystallographic notation for the univariant lattice complexes (e.g., I12xx for $\operatorname{Im} \overline{3} m(h), P n \overline{3} n(h)$, and $I 432(h)$ ) unsuitable since topology has no coordinate systems or coordinate; , thus we had to derive new notation for our on-line crystallographic orbifold atlas.[33].

We also combine the lattice complex information with the Wyckoff site and symmetry information and include non-characteristic invariant lattice complexes as well. For example, the entries for the Wyckoff sets in the Euclidean 3-orbifolds corresponding to the three space groups just mentioned become

\begin{tabular}{|l|c|c|l|l|}
\hline Mult. & Lat. Complex & Group Graph & Wyck. Set & Space G. \\
\hline $24-1$ & $\mathrm{I} 12\left[J_{2}\right] \mathrm{J}^{*} 4$ & $4^{\prime} 3^{\prime}<2^{\prime}>4^{\prime} 2^{\prime}$ & h:b-a & $\operatorname{Im} \overline{3} m(\mathrm{~h})$ \\
$24-1$ & $I 12\left[J_{2}\right] \mathrm{J}^{*} 4$ & $43<2>42$ & h:b-a & $\operatorname{Pn} \overline{3} n(\mathrm{~h})$ \\
$24-1$ & $\mathrm{I} 12\left[J_{2}\right] \mathrm{J}^{*} 4$ & $43<2>42$ & h:b-a & $I 432(\mathrm{~h})$ \\
\hline
\end{tabular}

The 24-1 is the Wyckoff multiplicity and number of equivalent Wyckoff sites in the Wyckoff set. In the lattice complex column, I12 and $\mathrm{J}^{*} 4$ are the invariant lattice complexes I and $\mathrm{J}^{*}$ at the ends of the univariant lattice complex, with Wyckoff multiplicity $1 / 12$ and $1 / 4$ that of the univariant site; $\left[J_{2}\right]$ is a non-characteristic lattice complex site half way between I and J, with Wyckoff multiplicity that of the univariant site. The next column gives the groups-on-graph description (described below) of the Wyckoff site, with the bracketed number indicating the order of the rotation axis $2^{\prime}(m m 2)$ or 2 -fold. The final column gives the univariant Wyckoff symbol, $h$, and that for the two invariant Wyckoff sites, $a$ and $b$, at the ends of $h$. 


\section{Crystallographic Orbifolds}

An orbifold [61] is a vector space modulo the symmetry group operating on that space. The crystallographic orbifolds $[12,13,29]$ of interest are $E^{3} / G, E^{2} / J$, and $S^{2} / K$. These are quotient groups of Euclidean 3-space, Euclidean 2-space, and the 2 -sphere modulo the 230 infinite space groups, $G, 17$ infinite plane groups, $J$, and 32 finite point groups, $K$, respectively. They are finite, closed, and usually singular, spaces called Euclidean 3-orbifolds, Euclidean 2-orbifolds and spherical 2-orbifolds, respectively. Singular set diagrams for the two sets of 2-orbifolds, and the 36 cubic 3-orbifolds are illustrated in our previous paper.[29] In addition, all orientable Euclidean 3-orbifolds, which includes those with underlying topological spaces, $S^{1} \mathrm{x}$ $S^{2}$ and several other lens spaces[47], are illustrated in Dunbar's thesis.[12]

\subsection{Underlying Topological Spaces}

Each Euclidean 3-orbifold has an underlying topological 3-space. The simplest are in the cubic crystal class, where the underlying spaces are $D^{3}, S^{3}, R P^{3}$, single $R P^{2}$ suspension, and double $R P^{3}$ suspension. (i.e., 3-disk, 3-sphere, real projective 3plane, real projective 2-plane plus a 3-disk half boundary, and double real projective plane, respectively).

1. $D^{3}$ : The 3-disk is a silvered 3-ball, so named because it has a mirror on its boundary and is a closed space since any vector from within which intersects the boundary is reflected back into the 3-ball.

2. $S^{3}$ : The 3-sphere is a 3-dimensional sphere embedded into a 4-dimensional Euclidean space, but which can be visualized as a Euclidean 3-space with a point at infinity. This is the next higher dimension analog of the projection of a 2-sphere onto a plane which is constructed by drawing a straight line from the north pole within the sphere to a point on the sphere, then continuing on that line until it intersects the plane tangential to the south pole of the sphere. The north pole itself projects to infinity in all directions in the plane, and is called the point at 2-infinity. To draw lines on the 3-sphere we take some topology artistic liberty and put the point at 3-infinity at some convenient nearby location in Euclidean 3-space and simply curve the relevant geodesic lines to go through that point (see orbifold F23 in Figure 5).

3. $R P^{3}: R P^{3}$ is a 3-dimensional projective plane embedded into a 4-dimensional Euclidean space. We cannot draw continuous lines of $R P^{3}$ in 3-dimensional Euclidean space, but we can use the antipodal 3-ball convention where any line that hits the boundary is transferred to the diametrically opposite point and reenters the antipodal 3-ball.

4. $R P^{2}$ suspension: An $R P^{2}$ suspension is visualized as a cone with apex at an orbifold point arising from a space group center of symmetry $(\overline{1}, \overline{4}$, or $\overline{3})$ which does not lie in a mirror. A 2-dimensional antipodal convention about the cone axis is used at the cone surface so that any line that intersects the cone surface from within is transferred 180 degrees about the cone axis and reenters. A dual suspension underlying space has two antipodal cones glued at their bases while a single suspension has a single cone with a silvered $D^{2}$ disk glued to its base. More complicated suspensions occur in orbifolds from the lower symmetry space groups, such as the one with eight antipodal cones from space group $P \overrightarrow{1}$. 


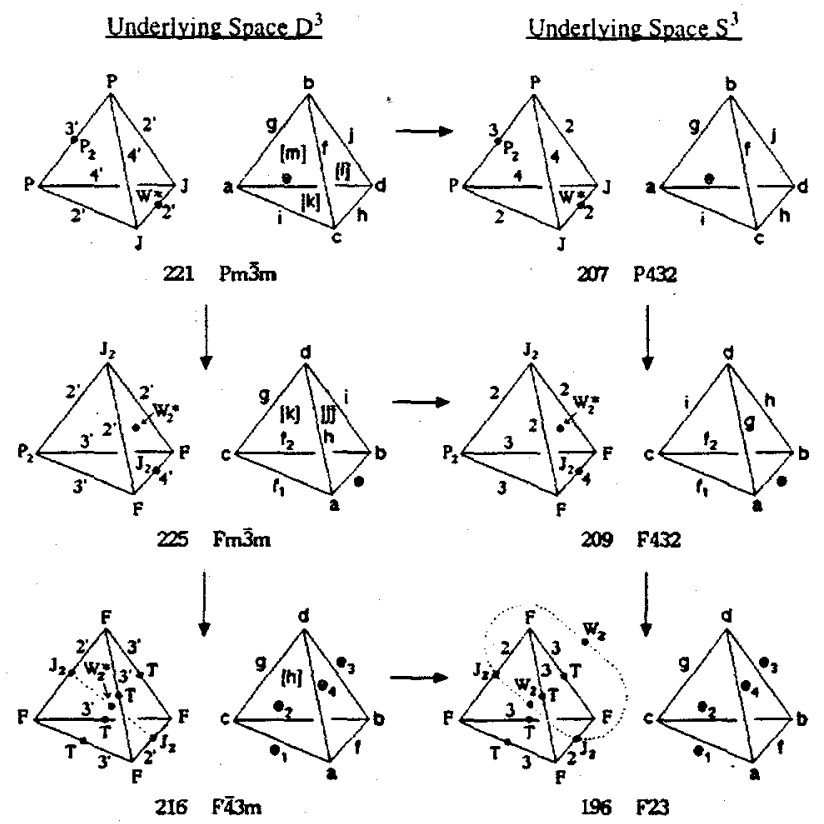

Figure 5: The Six Tetrahedral Euclidean 3-Orbifolds

\subsection{Spherical 2-orbifolds}

1. Singular Set Components: On an orbifold drawing, all mirrors and lines (other than construction lines) combine to form the singular set. Each of the singular set components (mirrors, lines, and line intersections) is one of the 32 spherical orbifolds with the name 1 for open space; $1^{\prime}$ for a mirror; 44 for a line labeled 4 ; 332 for the intersection of three lines labeled 3,3 , and $2 ; 4^{\prime} 3^{\prime} 2^{\prime}$ for intersection of lines $4^{\prime}, 3^{\prime}$, and $2^{\prime} ; 41^{\prime}$ when a 4 -axis meets a mirror; $23^{\prime}$ when a 2 -axis meets a $3^{\prime}$-axis; 0 for a stand-alone $R P^{2}$ cone point; 20 when a 2 -axis meets an $R P^{2}$ cone point; etc. See Johnson et al.[29] for a complete list and 2orbifold drawings.

2. Groups on Graph: The singular set of the orbifold forms a graph, and all components of the graph (i.e., nodes and links) are spherical orbifolds. A link between nodes is a subgroup of both nodes. The tetrahedron orbifold in $F m \overline{3} m$ (Figure 5 ) has $4^{\prime} 3^{\prime} 2^{\prime}, 3^{\prime} 3^{\prime} 2^{\prime}$, and $2^{\prime} 2^{\prime} 2^{\prime}$ nodes joined by $4^{\prime}-, 3^{\prime}$-, and $2^{\prime}$-fold links, respectively. The group graph symbols for the univariant lattice complexes are $3^{\prime} 2^{\prime}<4^{\prime}>3^{\prime} 2^{\prime}, 4^{\prime} 2^{\prime}<3^{\prime}>3^{\prime} 2^{\prime}, 4^{\prime} 3^{\prime}<2^{\prime}>2^{\prime} 2^{\prime}$, and $3^{\prime} 3^{\prime}<$ $\left.2^{\prime}\right\rangle 2^{\prime} 2^{\prime}$ with $\left\langle 2^{\prime}\right\rangle,\left\langle 3^{\prime}\right\rangle,\left\langle 2^{\prime}\right\rangle$, and $\left\langle 2^{\prime}\right\rangle$ the links. The $J_{2}\left[W_{2}^{*}\right] J_{2}$ univariant lattice complex in $F m \overline{3} m$ of Figure 5 (a link between a point at the center of the $4^{\prime} 4^{\prime}$ line and the $2^{\prime} 2^{\prime} 2^{\prime}$ vertex) is denoted $4^{\prime} 4^{\prime}<1^{\prime}>2^{\prime} 2^{\prime} 2^{\prime}$. This particular link is relevant in a later discussion. 
3. Knots, Links and Braids: Most of the Euclidean 3-orbifold singular sets are knotted graphs, but there is one pure knot, the figure eight singular set in the 3 -orbifold for cubic group $P 2_{1} 3$. There are several relatively simple links such as the Borromean rings[62] of the orthorhombic space group $I 2_{1} 2_{1} 2_{1}$ and the four link looped chain of $P 222_{1}[12]$. Braids are plentiful in the crystallographic 3 -orbifolds. $\{12,13]$

4. The Odd Axis Effect: Crystallographic groups have only one rotation axis of odd order, the 3-fold axis, and the rest are of order 2, 4 and 6 . Even and odd order functions often behave differently. In orbifold drawings, a Wyckoff $3^{\prime}$ site has different parts of the same Wyckoff mirror on the two sides of the $3^{\prime}$ axis, while even order axes do not. An intersection such as 332 has the same 3-axis coming in and going out, and a 322 intersection has the same 2-axis coming in and going out. In these cases we denote different parts of the same Wyckoff axis or mirror with subscripts. For example, note that the $F \overline{4} 3 m$ orbifold has one $3^{\prime}$ axis segmented into four parts and that a single mirror covers all four faces of this silvered 3-ball orbifold. McCullough et al.[43] discuss some aspects of this effect.

\subsection{Example 3-Orbifold Drawings}

The six cubic Euclidean 3-orbifolds with symmetry axes forming tetrahedra are illustrated in Figure 5. They have underlying spaces $D^{3}$ and $S^{3}$, as indicated, and are the simplest crystallographic 3-orbifolds. Each orbifold in Figure 5 has two drawings to minimize clutter in the labeling.

Left Figure: Capital letters indicate characteristic invariant lattice complex sites if on a vertex and non-characteristic invariant lattice complexes (defined below) if not on a vertex. The one digit numbers indicate rotation axis order and are primed if that axis lies on a mirror.

Right Figure: Small letter symbols are the Wyckoff letters used in ITCr-A [24] to identify specific Wyckoff sites. Letters at a vertex denote Wyckoff point sites, letters on an edge denote Wyckoff axis sites, and bracketed letters denote Wyckoff mirror sites.

Orbifold Atlas : A description of the 3-orbifold for $P m \overline{3} m$ follows. This entry is taken from our on-line world wide web orbifold atlas.[32] The $2^{*}$ in the bottom row of the group graph column denotes 2-fold pseudo-symmetry axis.

\begin{tabular}{|r|c|c|l|}
\hline Mult. & Lat. Complex & Group Graph & Wyckoff Set \\
\hline $1-2$ & $P$ & $4^{\prime} 3^{\prime} 2^{\prime}$ & $a, b$ \\
$3-2$ & $J$ & $4^{\prime} 2^{\prime} 2^{\prime}$ & $c, d$ \\
$6-2$ & $P 6[-] J 2$ & $3^{\prime} 2^{\prime}<4^{\prime}>2^{\prime} 2^{\prime}$ & $e: a-d, f: b-c$ \\
$8-1$ & $P 8\left[P_{2}\right] P 8$ & $4^{\prime} 2^{\prime}<3^{\prime}>4^{\prime} 2^{\prime}$ & $g: a-b$ \\
$12-1$ & $J 4\left[W^{*}\right] J 4$ & $4^{\prime} 2^{\prime}<2^{\prime}>4^{\prime} 2^{\prime}$ & $h: c-d$ \\
$12-2$ & $P 12[-] J 4$ & $4^{\prime} 3^{\prime}<2^{\prime}>4^{\prime} 2^{\prime}$ & $i: a-c, j: b-d$ \\
$24-2$ & & $m$ & $k: e h i, l: f h j$ \\
$24-2$ & & $m$ & $m 1: f g i, m 2: e g j$ \\
$48 .$. & & 1 & $n: k l m$ \\
$48-1$ & $P_{2} 6[-] W^{*} 4$ & $2^{*}=3^{\prime} 3^{\prime}<1>2^{\prime} 2^{\prime}$ & $n 1: g-h$ \\
\hline
\end{tabular}



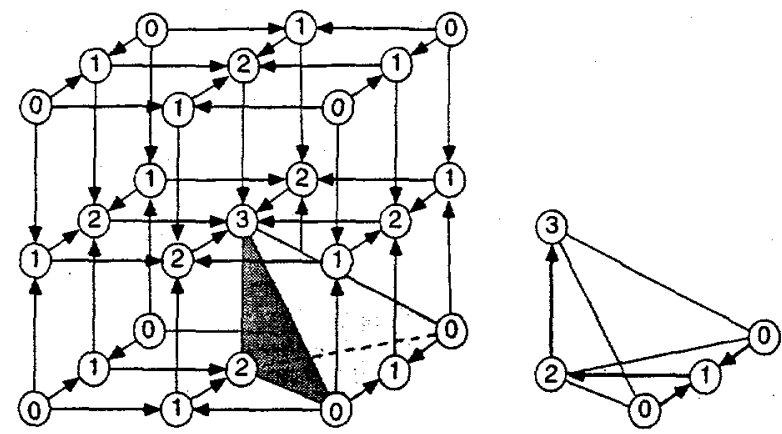

Figure 6: Sodium Chloride Critical Net (Fundamental Domain and 1/8 of Unit Cell shown)
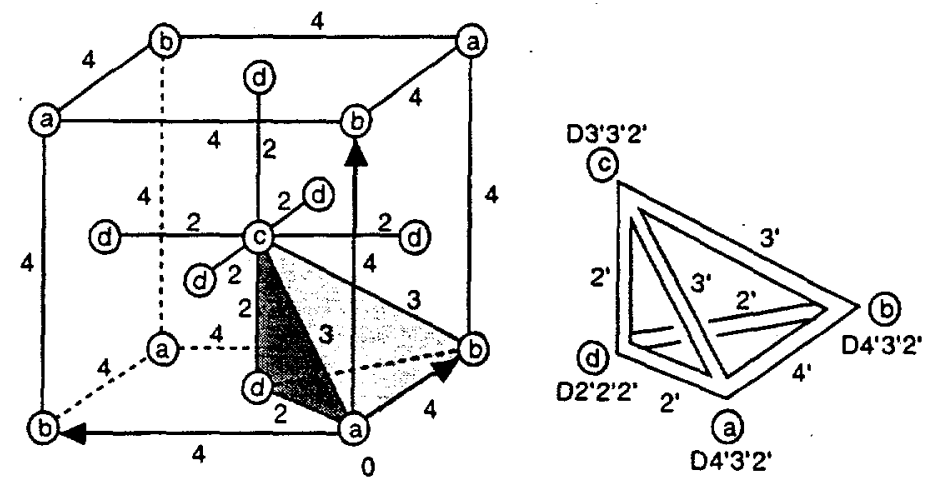

Figure 7: Sodium Chloride 3-Orbifold (1/8 of $F m \overline{3} m$ Unit Cell shown)

\section{Heegaard Splitting}

Our interest in Heegaard splitting stems from the fact that a Morse function (crystal structure) on a Euclidean 3-orbifold (i.e., wrapped up [orbi-folded] fundamental domain of a space group) is split into two parts by a level density surface, called the Heegaard surface, between the two types of saddle points.[51] This level surface has peaks and passes on one side, and pales and pits on the other. ${ }^{d}$

Since topologists have developed a number of techniques for characterizing Heegaard surfaces and transforming from one to another and crystallographers have several tens of thousands of crystal structures, each one of which has a different Heegaard surface, it seems worthwhile to study the Heegaard splitting literature to see what might be useful to crystallography. Here we will just make a few preliminary observations that could lead to more productive crystallographic applications. Compilations of data on small crystal structures, such as that by Hellner, Koch, and Reinhardt[25], should be useful in future research.

\footnotetext{
${ }^{d}$ Viewed from the standard crystallographic perspective, there are atoms and bonds on one side of the Heegaard surface and interstices on the other. This description reminds one of the minimal surface studies carried out by a number of crystallographers. [53] There are a growing number of topology papers $[48,20]$ comparing minimal surfaces and Heegaard surfaces.
} 


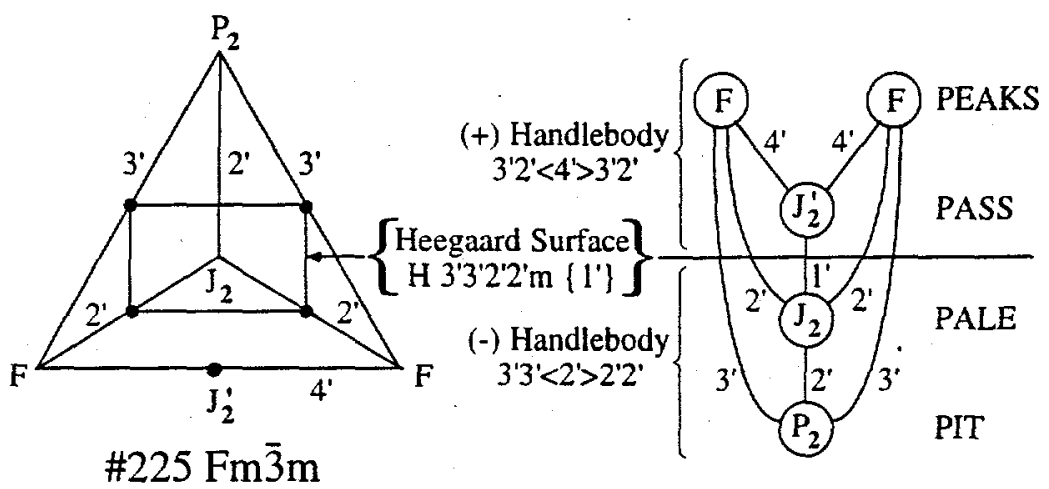

Figure 8: Heegaard Splitting of $\mathrm{NaCl}$ on $F m \overline{3} m$ 3-Orbifold

\subsection{Heegaard Splitting of $\mathrm{NaCl}$ Critical Net on Fm $\overline{3} m$ Orbifold}

Figure 6 shows the full critical net for a unit cell of $\mathrm{NaCl}$ (table salt), and Figure 7 shows the singular set for its space group. Both Figures 6 and 7 show a shaded fundamental domain of the unit cell, which for Figure 7 is also the 3-orbifold since it is bounded by mirrors. A detailed drawing of the $\mathrm{NaCl}$ Morse function is shown in Figure 4.

1. Critical Net Graph: The composite of the fundamental domains from Figures 6 and 7 can be stretched out in the direction of the arrows of Figure 6 by pulling up on the peaks and down on the pits to obtain the critical net graph on the right-hand side of Figure 8 with descending density sequence peaks, pass, pale, and pit. The corresponding orbifold for $F m \overline{3} m$ of Figure 5 is then placed on the left-hand side of Figure 7 , and the peak/pass/pale/pit lattice complex sequence $\left(F, F / J_{2}^{\prime} / J_{2} / P_{2}\right)$ from the left-hand orbifold drawing is placed within the nodes of the right-hand critical net graph. The prime on the first $J_{2}^{\prime}$ indicates it is a non-characteristic lattice complex site.

2. Heegaard Surface Nomenclature: The Heegaard surface cuts the tetrahedron orbifold parallel to the page on the left-hand drawing and cuts the distorted critical-net-on-orbifold perpendicular to the page on the right-hand drawing. Note that it cuts two $3^{\prime}$-axes, two $2^{\prime}$-axes, and the mirrors on the face of the 3 -orbifold, which produces the orbifold component $3^{\prime} 3^{\prime} 2^{\prime} 2^{\prime} m$ of the Heegaard surface symbol $H 3^{\prime} 3^{\prime} 2^{\prime} 2^{\prime} m\left\{1^{\prime}\right\}$. The critical net component $\left\{1^{\prime}\right\}$ of the symbol denotes that the Heegaard surface cuts a pass-to-pale link of the critical net, which is not an axis of the orbifold singular set. Pass-to-pale links are usually lower symmetry than the other links of the critical net. However, in those cases where all the pass-to-pale links are part of the singular set, we move that link to the curly brace. The $H$ in the symbol indicates it is a hyperbolic surface. The Heegaard surface is actually a 2 -orbifold, $[43,64,65]$ and we can calculate the Euler characteristic for a 2-orbifold[7] directly from the orbifold portion of the Heegaard surface symbol which gives $\chi<0$; thus, this Heegaard surface is a hyperbolic 2-orbifold. Most Heegaard surfaces are hyperbolic, but we also found a few that are Euclidean $\chi=0$, which we designate with a symbol starting with $E$ rather than $H$.

3. Handlebodies: The Heegaard surface, created by Heegaard splitting, parti- 
tions the manifold (or orbifold) into two handlebodies. A handlebody has a genus, $\mathrm{g}$ ( $\mathrm{g}=$ number of holes = number of handles), and is called a genus $\mathrm{g}$ handlebody. Both handlebodies from a Heegaard splitting have the same genus and a common boundary, the Heegaard surface. This means that all holes in the handlebodies go through the Heegaard surface. The handlebodies in Figure 8 are genus zero handlebodies since we are currently working with tetrahedral orbifolds. Since the 3-orbifold in Figure 8 has a mirror boundary, we should be using the term compression body[35] rather than handlebody, but for simplicity we will abuse topology terminology and use the term handlebody even when the orbifold has a (mirror) boundary.

4. Handlebody Spines: The review article, "Heegaard splitting of compact 3manifolds," by M. Scharlemann[51] discusses several approaches to Heegaard splitting. Since our main interest involves Morse functions, we will use the methods described in his section 2.4, "Splittings as Morse functions and as sweep-outs." Sweep-outs are based on the spines of the two handlebodies formed by the Heegaard splitting, and the spine $\Sigma$ of a handlebody $H$ is defined as the finite graph in $H$ to which $H$ deformation retracts. ${ }^{e}$ From our perspective, the deformation retract for $H_{+}$and $H_{-}$is simply those portions of the critical net graph above and below the Heegaard surface, respectively.

5. Handlebody Spine Orbifolds: McCullough et al.[43] and Zimmermann[64,65] call the orbifold singular set components in these deformation retracts handlebody orbifolds. For our application where we are adding the critical net of the Morse function to the orbifold, we could call them handlebody critical net orbifolds but prefer the shorter name handlebody spine orbifolds. In our on-line orbifold atlas, they are called simply handlebody orbifolds. The Euler constant for handlebody orbifolds can be calculated and some are tabulated. $[43,64,65]$

6. Handlebody Groups on Graphs: The $(+)$ and $(-)$ handlebody orbifolds of Figure 8 are $3^{\prime} 2^{\prime}<4^{\prime}>3^{\prime} 2^{\prime}$ and $3^{\prime} 3^{\prime}<2^{\prime}>2^{\prime} 2^{\prime}$, respectively. The unmatched links 3'3'2'2', the mirror $m$, and the pass to pale link $4^{\prime} 4^{\prime}<1^{\prime}>2^{\prime} 2^{\prime} 2^{\prime}$ combine to form the Heegaard surface symbol $H 3^{\prime} 3^{\prime} 2^{\prime} 2^{\prime} m\left\{1^{\prime}\right\}$.

\section{Quadrilateral Haken Normal Surfaces}

A tetrahedron has seven Haken normal surfaces, $[28,27]$ three of them are quadrilateral surfaces, such as the one shown in Figure 8, and the other four are triangular surfaces cutting the three edges extending from each vertex. Figure 9 shows the three quadrilateral surfaces in each of the three tetrahedral 3-orbifolds of Figure 5 with underlying space $D^{3}$. Crystal structures which have that quadrilateral Haken normal plane as a Heegaard surface are identified on the figure and described below.

\subsection{Normal Surface Automorphism}

The Euclidean 3-orbifolds from $F m \overline{3} m$ and $F \overline{4} 3 m$ have automorphisms, which are expressed as isomeric quadrilateral normal surface pairs corresponding to the crystal

\footnotetext{
'The definition of deformation retract in Chapter $O$ of Allen Hatcher's on-line book draft Algebraic Topology I at http://math.cornell.edu/hatcher is accompanied by an informative set of drawings.
} 


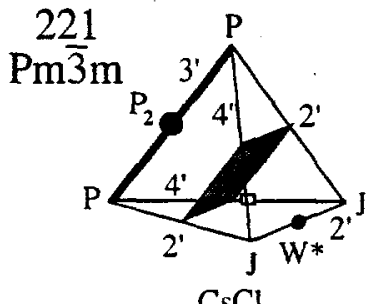

$\mathrm{CsCl}$ $\mathrm{H}^{\prime} \mathbf{2}^{\prime} 42^{\prime} \mathrm{\prime m}\{1\}$

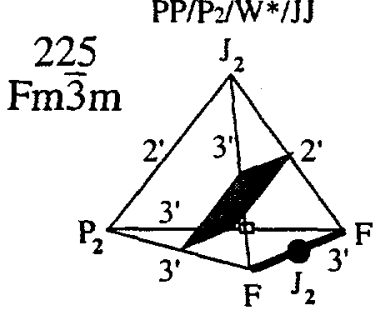

$\mathrm{NaCl}$

$\mathrm{H}^{\prime} 3^{\prime} 2^{\prime} 2^{\prime} \mathrm{m}\left\{\mathrm{l}^{\prime}\right\}$

$\mathrm{FF} / \mathrm{J}_{2} / \mathrm{J}_{2} / \mathrm{P}_{2}$

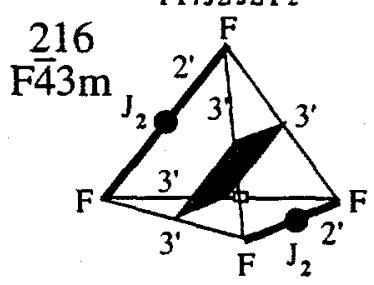

$\mathrm{NaCl}$

H3'3'3'3'm\{1\}

$\mathrm{FF} / \mathrm{J}_{2} / \mathrm{J}_{2} / \mathrm{FF}$

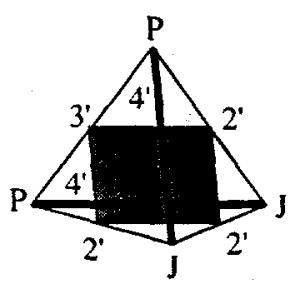

Simple Cubic

$\mathrm{H}^{\prime} 2^{\prime} 2^{\prime} \mathrm{m}\left\{2^{\prime}\right\}$ $\mathrm{P} / \mathrm{J} / \mathrm{J} / \mathrm{P}$
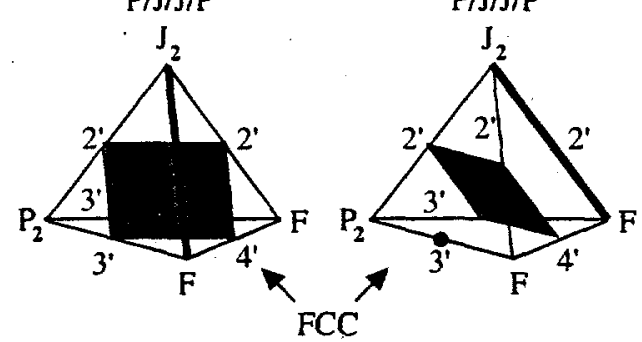

$\mathrm{H}^{\prime} 3^{\prime} 2^{\prime} \mathbf{2}^{\prime} \mathrm{m}\left\{\mathrm{l}^{\prime}\right\}$

$\mathrm{F} / \mathrm{J}_{2} / 3 / \mathrm{FP}_{2}$
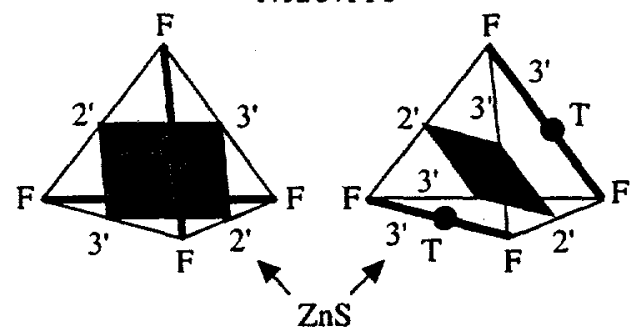

H3'2'3'2'm $\{1\}$

$\mathrm{FF} / \mathrm{T} / \mathrm{T} / \mathrm{FF}$

Figure 9: Normal Quadrilateral Haken Surfaces for Three Tetrahedral Cubic Orbifolds

structure types face centered cubic (FCC) and zinc sulfide, respectively, as shown in Figure 9. The dual (reversed critical point sequence) of FCC is the $\mathrm{Li}_{2} \mathrm{O}$ (lithium oxide) structure shown in Figure 3 , which is perhaps a better default characterization than FCC because there is only one pit for $\mathrm{Li}_{2} \mathrm{O}$ and two for FCC. However, the fact that there are so many FCC metals makes it a more familiar term to most scientists.

At first glance one might also expect $P m \overline{3} m$ to have a pair of isomorphic quadrilateral normal surfaces since two of them have the same critical point sequence of lattice complexes, $\mathrm{P} / \mathrm{J} / \mathrm{J} / \mathrm{P}$. However the descriptors for those two quadrilateral normal surfaces are $3^{\prime} 2^{\prime} 2^{\prime} 2^{\prime}$ and $4^{\prime} 3^{\prime} 4^{\prime} 2^{\prime}$ so they cannot be isomorphic. Only the first of these two is a valid Heegaard surface ${ }^{f}$ To see why the quadrilateral normal surface $3^{\prime} 2^{\prime} 2^{\prime} 2^{\prime}$ of $P m \overline{3} m$ is a valid Heegaard surface while $4^{\prime} 3^{\prime} 4^{\prime} 2^{\prime}$ is not, we use the wagon wheel axle $\mathrm{Q}$ rule of section 4.4 to calculate $Q_{1}=Q_{2}=2$ for the former and $Q_{1}=Q_{2}=4$ for the latter. The critical nets are $3^{\prime} 2^{\prime}<4^{\prime}>2^{\prime}<2^{\prime}>2^{\prime}<4^{\prime}>3^{\prime} 2^{\prime}$, and $4^{\prime} 3^{\prime}<2^{\prime}>2^{\prime}<4^{\prime}>2^{\prime}<2^{\prime}>4^{\prime} 3^{\prime}$ which have critical point and separatrix-line Wyckoff multiplicities $1(6) 3(12) 3(6) 1$ and $1(12) 3(6) 3(12) 1$, respectively. Thus $Q_{1}$ $=Q_{2}=6 / 3=2$ for the former and $Q_{1}=Q_{2}=12 / 3=4$ for the latter. All adjacent 
multiplicity ratios are in fact useful. For the (valid) first series we see that there are $6 / 1=6$ passes around each peak, $6 / 3=2$ peaks around each pass, $12 / 3=4$ pales around each pass, $12 / 3=4$ passes around each pale, $6 / 3=2$ pits around each pale, and $6 / 1=6$ pales around each pit. Thus the wagon wheels around the peak-passpeak axis (and pit-pass-pit axis) have 4 spokes. The corresponding structure type is called simple cubic. The only known real crystal with this structure is $\alpha$-polonium (Po, atomic number 84 ).

\subsection{Symmetry Breaking Subgroup Sequence}

Note that there are two $\mathrm{NaCl}$ structures in Figure 9. In addition, the simple cubic structure is geometrically very similar to $\mathrm{NaCl}$. Thus we have three related structures on a series of normal subgroups of index two. The highest symmetry structure, simple cubic $P m \overline{3} m$, is changed to a larger face centered cell, with $P$ and $J$ going to $P_{2}$ and $J_{2}$. The larger primitive cell changes to a face centered cell by making adjacent peaks alternate between $\mathrm{Na}$ and $\mathrm{Cl}$. Thus the old peak $P$, now $P_{2}$, splits into two $F s$ (using the lattice complex identity $P_{2}=F+F "$ ) and the old pass, now $J_{2}$, becomes a non-characteristic $J_{2}$ in the new space group $F m \overline{3} m$.

For the change from $F m \overline{3} m$ to $F \overline{4} 3 m$, we split the remaining $P_{2}$ site into two $F$ sites (using the lattice complex identity $P_{2}=F+F^{\prime \prime}$ ) and turn the remaining $J_{2}$ characteristic lattice complex site into a $J_{2}$ non-characteristic site, to form $F \overline{4} 3 m$. The final space group may at first seem to be higher symmetry since it has $4 F$ invariant lattice complex sites, but these are normalizer (automorphism) equivalent sites, not symmetry equivalent sites. For a more graphic illustration of symmetry breaking, see the cubic group/subgroup appendix and series of critical net family diagrams in our previous paper.[29]

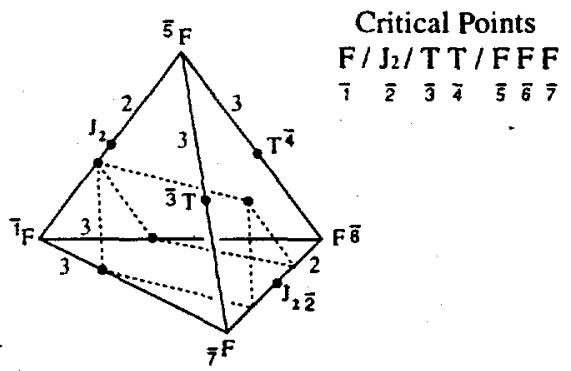

$$
\begin{aligned}
& 32<3>2<3>32(-) \text { handlebody } \\
& \text { H33222 }\{11\} \quad \text { Heegaard surface } \\
& 332<1>22
\end{aligned}
$$

Figure 10: Heegaard Splitting of FCC Critical Net on F23 3-Orbifold

\subsection{Normal Surfaces in Triangulated Orbifolds}

Figure 10 illustrates the non-planar Heegaard surface in the body centered cubic structure, which can be represented by two Haken normal surface tetrahedra. This process is the foundation of Haken normal surface (and almost normal surface) analysis. The mathematical description of an arbitrary Heegaard surface in terms of a large number of tetrahedra from a fine triangulation of an orbifold can present nontrivial mathematical problems because of the integer nature of the equations involved, but this is currently a very active research area. 


\subsection{Heegaard Surfaces in Orbifolds with $R P^{2}$ Suspension Underlying Space}

Certain crystal structure types, particularly those in the body centered cubic (BCC) and diamond families, occur on space groups having 3-orbifolds with an $R P^{2}$ suspension as the underlying space. An example is the BCC critical net on the $P n \overline{3}$ 3-orbifold shown in Figure 11. One must be careful in the analysis of projective spaces because the complete interior is unique and only the surface has the antipodal property described previously. Note that the $W^{*}$ lattice complex point is non-characteristic; thus the 2-axis out of the characteristic $J^{*}$ loops back to itself and the $(-)$ handlebody is denoted $2<2>\&$. Consequently, $W^{*}$ is in the middle of the 2-axis loop and the univariant lattice complex symbol for that handlebody becomes $J * 2[W *] \&$.

\subsection{Triply Periodic Minimal Surface}

Figure 12 shows the Heegaard surface for a unit cell of the simple cubic structure when the properly curved fundamental domain is repeated 48 times using the full symmetry of the $P m \overline{3} m$ space group. Figure 12 is actually Schwartz's triply periodic minimal surface[20] P, but the Heegaard level surface closely approximates it. The full surface requires gluing such units together into a three dimensional repeating array. This is a demonstration figure for Brakke's Surface Evolver program.[5] The heavy lines are mirrors of $P m \overline{3} m$ which clearly outline the $H 3^{\prime} 2^{\prime} 2^{\prime} 2^{\prime} m$ surface motifs. The program seems capable of making such drawings for all the Heegaard surface discussed above.

\section{Heegaard Surface Kinship}

The crystal structure types appearing in Figure 8 represent surprisingly diverse major crystal chemistry families which develop increasingly complex Heegaard surfaces as the number of different atoms increases. Terms like class, family, etc. are already so heavily overused and ambiguous that it is hard to find a suitable traditional scientific term for a crystallographic Heegaard surface classification. We have considered terms like kin and kinship but need more experience with Heegaard surfaces and a better feel for their usefulness before worrying about considering a classification.

A list of Heegaard surfaces directly related to and including the ones shown above is included as the Appendix. The BCCs clearly show that Heegaard surfaces

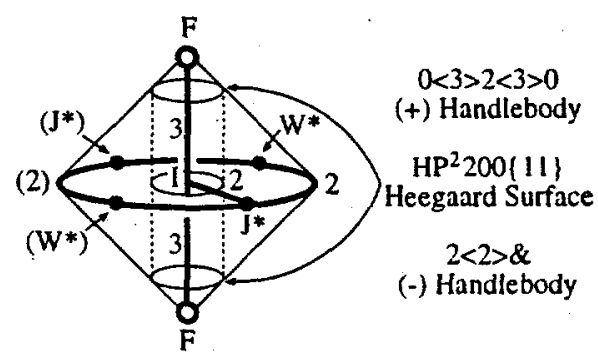

$$
\begin{gathered}
\text { Antipodal Cone } \\
\text { Double Suspension } \\
{\left[W^{*}=\left(W^{*}\right), J^{*}=\left(J^{*}\right),\right.}
\end{gathered}
$$

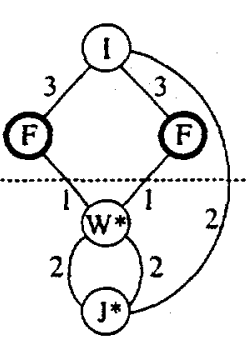

Critical Net on Orbifold

Figure 11: Heegaard Splitting of BCC Critical Net on $P n \overline{3}$ 3-Orbifold 


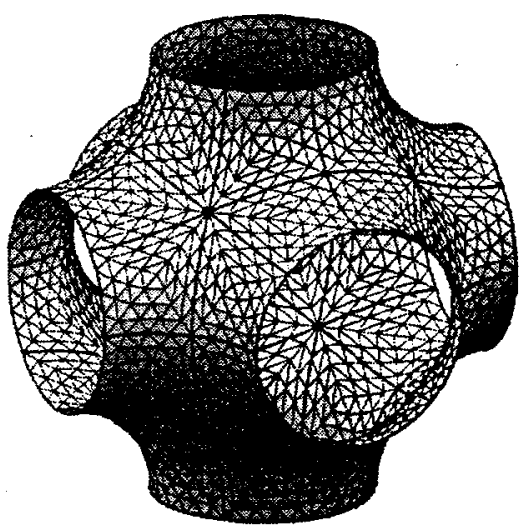

Figure 12: Simple-Cubic Heegaard Surface Approximates Schwartz's P Surface (A Triply Periodic Minimal Surface)

alone do not uniquely specify a crystal structure since there are a number of duplicates in that group. Most of the data in the appendix are easily derived from the critical net graphs in our first paper.

\section{Whither and Yon}

We routinely use "whither and yon" notebooks (to what distant place and beyond) to periodically record our aspirations for future research. Our previous paper has a section called "Where do we go from here?" which contains a number of research needs in crystallographic topology, and we add a few additional suggestions below.

\subsection{Heegaard Splitting}

Heegaard transformations: The mathematical literature on transforming Heegaard surfaces is very convoluted and time consuming to understand. Some review articles for the layman would indeed be welcome. At present our understanding suggests that although normal surfaces seem to work, the mathematical problems in solving the required integer equations seem too formidable for the large scale problems which could develop in crystallography. We are currently putting our efforts into understanding the Rubinstein-Scharlemann Graphic approach $[49,50,39]$ which is based on Cerf theory and uses a smooth manifold topology approach, rather than piecewise linear topology. This method provides a systematic procedure for comparing two Heegaard surfaces.

Handlebody nomenclature: It seems advisable to use the handlebody orbifold notation of Zimmermann[65] which records the routing of connections between the two handlebodies as a braid to provide a more complete description of the singular set topology.

Invariants: The problem with most mathematical invariants is that they are designed to prove theorems rather than provide physical information. However, invariants such as the Heegaard numbers described by Zimmermann and others may provide useful information if someone can give them a physical interpretation. 


\subsection{Fundamental Group of an Orbifold}

For orbifolds to be useful in crystallography, they have to apply equally to all space groups. There are 10 spacegroups (13 if handedness is included) which orbi-fold into Euclidean 3-manifolds, which have no singular set. Thus their orbifold drawing would be a blank figure. The Euclidean manifolds put all their symmetry into the fundamental group.[34] On the other extreme, the symmorphic space groups form simply connected 3-orbifolds with trivial fundamental group and an extensive singular set. The other space groups lie between these two extremes. Thus we need some way of adding the fundamental group to the orbifold representation. Unfortunately, fundamental groups often involve group presentation methods that many physical scientists find not useful.

Stated another way, we need some way of representing screw axes and glide planes, in an extended orbifold, as easily as we now show mirrors, rotation axes, and inversion centers. These features are all shown in the ITCr space group drawings.[24] In orbifold drawings, some screws axes show up indirectly in the helix core of a twisted pair of rotation axes, but that is not a consistent representation.

Seifert fibered Euclidean 3-orbifolds may be generated by lifting from base Euclidean 2-orbifolds[6]. Although that approach does provide information (from obstructions) suitable for a rather awkward nomenclature[8] system for a large subset of the Euclidean 3-orbifolds, that approach does not work for the cubic space groups since the 3 -axis through the body diagonal of the cubic interchanges fibration along the cube edges.

\subsection{Stochastic Thermal Motion Analysis}

Though the thermal ellipsoids of structural crystallography contain large quantities of information about thermal motion in crystals, they are usually published without interpretation. A valid stochastic thermal motion analysis method is needed that does not require extensive dynamic or mechanistic modeling for each new crystal structure. It will be interesting to see how far the Radon-Nikodym property can be taken in meeting that goal.

\subsection{Category Theory of Crystallographic Topology}

The paper, "Orbifolds, Sheaves and Groupoids", by Moerdijk and Pronk[44] and Pronk's thesis[45], "Groupoid Representations for Sheaves on Orbifolds" show that orbifolds correspond exactly to a specific class of smooth groupoids, and that each such groupoid determines a category of equivariant sheaves. Expressing orbifolds in these terms opens the possibility of reformulating all the primitives of crystallographic topology into categories $[41,42]$ which could provide even broader inroads for crystallographic utilization of contemporary mathematical techniques

All the techniques described in the present paper might be classified as "bottom up" approaches while category theory is basically a "top down" approach. For many research problems it is highly desirable to be able to use both approaches. If a problem seems intractable using the traditional techniques of that discipline, it is sometimes possible to transform that problem to look like a completely different problem, which has known solutions, by using category of categories homeomorphisms. This would not be a short term research project, but nevertheless it seems to us both feasible and highly desirable. 


\section{Acknowledgments}

I gratefully acknowledge the assistance from my topology coworkers. From 1993 until my retirement in 1996, Bill Dunbar, Peter Brinkmann, and Jim Davis patiently continued to explained the intricacy of topology until I finally started to understand some of it. Klaus Johannson of the University of Tennessee, Knoxville continues to show me the beauty of topology and supplies invaluable advice on numerous occasions. My special thanks go to Mike Burnett for his warm friendship and close professional collaboration during the past 15 years.

Post retirement continuation of the research is made possible by an understanding spouse, the other Carol Johnson, and the kind assistance of the Chemical and Analytical Sciences Division in providing retiree space and computing facilities at Oak Ridge National Laboratory.

Research sponsored by the Laboratory Directed Research and Development Program of the Oak Ridge National Laboratory, managed by Lockheed Martin Energy Research Corp. for the U.S. Department of Energy under Contract No. DE-AC05-96OR22464.

U.S. GOVERNMENT PURPOSES NOTICE

"The submitted manuscript has been authored by a contractor of the U.S. Government under contract No. DE-AC05-960R22464. Accordingly, the U.S. Government retains a nonexclusive, royalty-free license to publish or reproduce the published form of this contribution or allow others to do so, for U.S. Government purposes." 
Appendix - Cubic Crystal Structure Heegaard Surfaces

The line $\left(203-F d \overline{3}-D / T / T / D-H P^{2} 3200\{1\}\right)$ signifies space group number 203 (ITCr number), with peak/pass/pale/pit invariant lattice complexes critical points $D / T / T / D$, and hyperbolic Heegaard surface $H P^{2} 320\{1\} . H P^{2}$ in the Heegaard surface symbol denotes the hyperbolic surface lies in an underlying topological space with a single suspension projective plane $R P^{2}$. The double suspension is denoted by two zeros $(00)$ in the hyperbolic plane symbol.

\begin{tabular}{|c|c|c|c|c|}
\hline Struc. & ITCr & Sp. Gr. & Critical Points & Heegaard Surface \\
\hline \multirow{7}{*}{ SCube } & 221 & $P m \overline{3} m$ & $P / J / J / P$ & $H 3^{\prime} 2^{\prime} 2^{\prime}\left\{2^{\prime}\right\}$ \\
\hline & 207 & $P 432$ & $P / J / J / P$ & $H 322\{2\}$ \\
\hline & 200 & $P m 3$ & $P / J / J / P$ & $H 3 m\left\{2^{\prime} 2^{\prime}\right\}$ \\
\hline & 215 & $P \overline{4} 3 m$ & $P / J / J / P$ & $H 3^{\prime} 3^{\prime} m\{2\}$ \\
\hline & 226 & $F m \overline{3} c$ & $P_{2} / J_{2} / J_{2} / P_{2}$ & $H 32 m\left\{1^{\prime}\right\}$ \\
\hline & 195 & $P 23$ & $P / J / J / P$ & $H 33\{22\}$ \\
\hline & 219 & $F \overline{4} 3 c$ & $P_{2} / J_{2} / J_{2} / P_{2}$ & $H 3300\{1\}$ \\
\hline \multirow[t]{4}{*}{$\mathrm{NaCl}$} & 225 & $F m \overline{3} m$ & $F F / J_{2} / J_{2} / P_{2}$ & $H 3^{\prime} 3^{\prime} 2^{\prime} 2^{\prime} m\left\{1^{\prime}\right\}$ \\
\hline & 209 & $F 432$ & $F F / J_{2} / J_{2} / P_{2}$ & $H 3322\{1\}$ \\
\hline & 202 & $F m \overline{\mathbf{3}}$ & $F F / J_{2} / J_{2} / P_{2}$ & $H 33 m\{1\}$ \\
\hline & 229 & $I m \overline{3} m$ & $I J^{*} / 4^{\prime} W^{*} / J_{2} / P_{2}$ & $H 3^{\prime} 2^{\prime} 2^{\prime} 2^{\prime} 2 m\left\{1^{\prime} 1^{\prime}\right\}$ \\
\hline \multirow[t]{2}{*}{$\mathrm{NaCl}+$} & 216 & $F \overline{4} 3 m$ & $F F / J_{2} / J_{2} / F F$ & $H 3^{\prime} 3^{\prime} 3^{\prime} 3^{\prime} m\{1\}$ \\
\hline & 196 & $F 23$ & $F F / J_{2} / J_{2} / F F$ & $H 3333\{1\}$ \\
\hline \multirow[t]{12}{*}{$\overline{B C C}$} & 229 & $I m 3 m$ & $I / P_{2} / W^{*} / J^{*}$ & $H 4^{\prime} 2^{\prime} m\{2\}$ \\
\hline & 432 & $I 432$ & $I / P_{2} / W^{*} / J^{*}$ & $H 42\{22\}$ \\
\hline & 204 & $\operatorname{Im} \overline{\mathbf{3}}$ & $I / P_{2} / W^{*} / J^{*}$ & $H P^{2} 2^{\prime} 0 m\{1\}$ \\
\hline & 217 & $I \overline{4} 3 m$ & $I / P_{2} / W^{*} / J^{*}$ & $H P^{2} 2^{\prime} 0 m\{1\}$ \\
\hline & 222 & $P n \overline{3} n$ & $I / P_{2} / W^{*} / J^{*}$ & $H P^{2} 420\{1\}$ \\
\hline & 224 & $P n \overline{3} m$ & $I / F F / W^{*} / J^{*}$ & $H 2^{\prime} m\{22\}$ \\
\hline & 223 & $P m \overline{3} n$ & $I / P_{2} / W W / J^{*}$ & $H 2^{\prime} m\{22\}$ \\
\hline & 197 & $I 23$ & $I / P_{2} / W^{*} / J^{*}$ & $H P^{3} 200\{11\}$ \\
\hline & 201 & $P n \overline{3}$ & $I / F F / W^{*} / J^{*}$ & $H P^{2} 200\{11\}$ \\
\hline & 208 & $P 4_{2} 32$ & $I / F F / W W / J^{*}$ & $H 2\{2222\}$ \\
\hline & 218 & $P \overline{4} 3 n$ & $I / P_{2} / W W / J^{*}$ & $H P^{2} 200\{11\}$ \\
\hline & 228 & $F d \overline{3} c$ & $I_{2} / F_{2} F_{2} / W_{2}^{*} / J_{2}^{*}$ & $H P^{2} 20\{221\}$ \\
\hline $\mathrm{CsCl}$ & 221 & $P m \overline{3} m$ & $P P / P_{2} / W^{*} / J J$ & $H 4^{\prime} 2^{\prime} 4^{\prime} 2^{\prime} m\{1\}$ \\
\hline \multirow[t]{3}{*}{$\overline{\mathrm{FCC}}$} & 225 & $F \bar{m} \bar{m}$ & $F \bar{P}_{2} / 3^{\prime} / J_{2} / F$ & $H 4^{\prime} 3^{\prime} 2^{\prime} 2^{\prime} m\left\{1^{\prime}\right\}$ \\
\hline & 209 & $F 432$ & $F P_{2} / 3 / J_{2} / F$ & $H 4322\{1\}$ \\
\hline & 202 & $F m \overline{3}$ & $F P_{2} / 3 / J_{2} / F$ & $H 322^{\prime} m\{1\}$ \\
\hline \multirow[t]{2}{*}{$\mathrm{FCC}+$} & 216 & $F \overline{4} 3 m$ & $F F F / T T / J_{2} / F$ & $H 3^{\prime} 2^{\prime} 3^{\prime} 2^{\prime} 2^{\prime} m\left\{1^{\prime} 1^{\prime}\right\}$ \\
\hline & 196 & $F 23$ & $F F F / T T / J_{2} / F$ & $H 33222\{11\}$ \\
\hline \multirow[t]{3}{*}{ Diam } & $\overline{227}$ & $\overline{F d} \overline{3 m}$ & $\bar{D} / T / T / D$ & $H 3^{\prime} 2^{\prime} m\{2\}$ \\
\hline & 210 & $F 4_{1} 32$ & $D / T / T / D$ & $H 32\{22\}$ \\
\hline & 203 & $F d \overline{\mathbf{3}}$ & $D / T / T / D$ & $H P^{2} 3200\{1\}$ \\
\hline \multirow[t]{2}{*}{$\mathrm{ZnCl}$} & 216 & $F \overline{4} 3 m$ & $F F / T / T / F F$ & $H 3^{\prime} 2^{\prime} 3^{\prime} 2^{\prime} m\{1\}$ \\
\hline & 196 & $F 23$ & $F F / T / T / F F$ & $H 3232\{11\}$ \\
\hline \multirow[t]{3}{*}{$\mathrm{NaTl}$} & 227 & $F d 3 m$ & $D D / T F_{2} T / W_{2}{ }^{*} / J_{2}{ }^{*}$ & $H 2^{\prime} 2^{\prime} m\{221\}$ \\
\hline & 210 & $F 4_{1} 32$ & $D D / T F_{2} T / W_{2} W_{2} / J_{2}{ }^{*}$ & $E 22\{2211\}$ \\
\hline & 203 & $F d \overline{\mathbf{3}}$ & $D D / T F_{2} T / W_{2}{ }^{*} / J_{2}^{*}$ & $E P^{2} 220\{11\}$ \\
\hline
\end{tabular}




\section{References}

1. M.A. Akivis and V.V. Goldberg, Projective Differential Geometry of Submanifolds, North Holland, Amsterdam, 1993.

2. E. Ascher and A. Janner,Helv. Phys. Acta 38, 551-572, 1965.

3. W.R. Busing and H.A. Levy, Acta Cryst.17, 142, 1964.

4. R.D. Bourgin, Geometric Aspects of Convex Sets with the Radon-Nikodym Property, Lect. Notes in Math. 993, Springer-Verlag, Berlin, 1983.

5. K. Brakke, The Surface Evolver. Experimental Math. 1 141-165, 1992.

6. F. Bonahon and L. Siebenmann, "The Classification of Seifert Fibered 3Orbifolds" in Low Dimensional Topology, Cambridge University Press, pp. 19-85, 1985.

7. J.H. Conway, "The Orbifold Notation for Surface Groups in Groups", Combinatorics and Geometry, M. Liebeck and J. Saxl, eds, Cambridge University Press, pp. 438-447, 1992.

8. J.H Conway and W.T Thurston, "Two and Three-Dimensional Euclidean Orbifold Voodoo", preprint, 1996.

9. P. Coppens, "The Structure Factor", International Tables for Crystallography Volume B: Reciprocal Space Second Edition, Editor: U. Shmueli, 1996.

10. A.W.M. Dress, D.H. Huson, and E. Molnar. "The classification of facetransitive 3d-tilings". Acta Crystallographica, A49:806-817, 1993.

11. J. Diestel and J.J. Uhl, Jr., Vector Measures, Math. Surveys 15, Amer. Math. Soc., Providence, R.I., 1977.

12. W.D. Dunbar, Fibered Orbifolds and Crystallographic Groups. Ph.D. Dissertation, Princeton Univ., 1981.

13. W.D. Dunbar, "Geometric Orbifolds", Rev. Mat. Univ. Comp. Madrid, 1, $67-99,1988$.

14. M.N. Burnett and C.K. Johnson, ORTEP-III: Oak Ridge Thermal Ellipsoid Plot Program for Crystal Structure Illustrations, Oak Ridge Nat. Lab. Rep. ORNL-6895, 1996.

15. P. Engel, T. Matsumoto, G. Steinmann and H. Wondratschek. The Noncharacteristic Orbits of the Space Groups, Supp. Issue No. 1, Zeits. fur Krist, Oldenbourg Verlag, Munchen., 1984.

16. D.R. Farkas, "Crystallographic Groups and their Mathematics", Rocky Mount. J of Math, 11 511-551, 1981.

17. W. Fisher, H. Burzlaff, E. Hellner, and J.D.H. Donnay, Space Groups and Lattice Complexes, Nat. Bur. of Standards Mono. 134, U.S. Gov. Print. Off., Wash. D.C., 1973.

18. W. Fisher and E. Koch, Kubische "Struckturtypen mit Festen Koordinates", Zeit. fur Krist, 140, 324-330, 1974.

19. O.D. Friedrichs and D.H. Huson, "Orbifold triangulations and crystallographic groups". Per. Math. Hung, 34(1-2):29-55, 1997.

20. C. Frohman and W.H. Meeks III, "The Topological Uniqueness of Complete One-Ended Minimal Surfaces and Heegaard Surfaces in $R^{3}$ ", J. Amer. Math. Soc, 10, 495-512, 1997.

21. M. Goresky and R MacPherson, Stratified Morse Theory Springer-Verlag, New York, 1988.

22. E.H. Grosse, Approximation and Optimization of Electron Density Maps, Ph. D. Dissertation, Stanford Univ., 1980.

23. I.I, Gihman and A.V. Skorohod, The Theory of Stochastic Processes I, Springer-Verlag, New York, NY, pp 496, 1974. 
24. Th. Hahn, ed., International Tables for Crystallography, Volume A: SpaceGroup Symmetry, Klewer, Boston, 1992

25. E. Hellner, E. Koch, and A. Reinhardt, "The Homogeneous Frameworks of the Cubic Crystal Structures", Physics Data NR 16-2 Fact Informations-Zentrum, Alle Rechte Vorbehalten pp 1-67, 1981.

26. K. Ito, ed., Encyclopedic Dictionary of Mathematics by the Mathematical Society of Japan, Second Ed., MIT Press, Cambridge, Mass., 1985.

27. W. Jaco and E. Sedgwick, "Decision Problems in the Space of Dehn Fillings", preprint math.GT/9811031, 1998.

28. K. Johannson. Topology and Combinatorics of 3-Manifolds, Lect. Notes in Math. 1599, Springer-Verlag, Berlin, 1995.

29. C.K. Johnson, M.N. Burnett and W.D. Dunbar, "Crystallographic Topology and its Applications", Crystallographic Computing 7 Proceedings from the Macromolecular Crystallography Computing School, Eds. P. E. Bourne and $\mathrm{K}$. Watenpaugh, University Press (in press).

30. C.K. Johnson, "Generalized Treatments for Thermal Motion", Thermal Neutron Diffraction, Ed. B.T.M Willis, Oxford Univ. Press pp 132-160, 1970.

31. C.K. Johnson, "Stochastic Thermal Motion Analysis and the Radon-Nikodym Property". Abstracts of the Amer. Cryst. Assoc, Buffalo Meeting, 1999.

32. C.K. Johnson and M.N. Burnett. Orbifold Atlas, Crystallographic Topology Web Site, 1999. http://www.ornl.gov/ortep/topology.html

33. C.K. Johnson and M.N. Burnett. "Heegaard Splitting of Critical Nets on Orbifolds", Abstracts of Amer. Cryst. Assoc, Washington DC Meeting, 1998.

34. J.F. Kennison, "What is the Fundamental Group?" J. Pure Appl. Algebra, $59,187-200,1989$.

35. J. Kalliongis and D.McCullough, "Orientation-Reversing Involutions on Handlebodies", Trans. Amer. Math. Soc, 348, 1739-1755, 1996.

36. E. Koch, "Die Grenzformen der Kubischen Gitterrkomplexe", Zeit. Krist, 140, 75-86, 1974.

37. E. Koch, "A Geometric Classification of Cubic Point Configurations". Zeit. Krist, 166, 23-52, 1984.

38. E. Koch and W. Fischer, "Lattice Complexes and Limiting Complexes versus Orbit Type and Non-characteristic Orbits: A comparative Discussion", Acta Cryst, A41, 421-426, 1985.

39. T. Kobayaski and O. Saeki, "Rubinstein-Scharlemann Graphic of 3-Manifold as the Discriminant Set of a Stable Map", preprint, 1998.

40. M. Ludoux and M. Talagrand. Probability in Banach Spaces: Isoperimetry and Processes, Springer-Verlag, Berlin, 1991.

41. S. MacLane, Categories for the Working Mathematician, Springer, 1999.

42. S. MacLane and I. Moerdijk, Sheaves in Geometry and Logic: A First Introduction to Topos Theory Springer, 1992.

43. D. McCullough, A. Miller, and B. Zimmerman, "Group actions on Handlebodies", Proc. London Math. Soc, 59 373-416, 1989.

44. I. Moerdijk and D.A. Pronk, "Orbifolds, Sheaves and Groupoids", K-theory 12, 3-21, 1997.

45. D.A. Pronk, Groupoid Representations for Sheaves on Orbifolds, $\mathrm{PhD}$ Thesis, Utrecht Univ., Netherlands, 1995.

46. M. Richter. Approximation of Gaussian Random Elements and Statistics, Teubner, Stuttgart, pp 44, 1992.

47. D. Rolfsen, Knots and Links, Publish or Perish, Inc., Houston, TX, 1990. 
48. J.H. Rubinstein, "Polyhedral Minimal Surfaces, Heegaard Splittings and Decision Problems for 3-dimensional Manifolds", AMS/IP Studies in Adv. Math. 2, (Part 1) 1-20, 1997.

49. H. Rubinstein and M. Scharlemann, "Comparing Heegaard Splittings of nonHaken 3-manifolds", (1996), Topology, 35, 1005-1026, 1996.

50. H. Rubinstein and M. Scharlemann, "Comparing Heegaard Splittings - the Bounded Case", Trans. Amer. Math. Soc, 350, 689-715, 1998.

51. M. Scharlemann, "Heegaard Splittings of Compact 3-Manifolds", in Handbook of Geometric Topology, ed. by R. Daverman and R. Sher, Elsevier, (to appear).

52. E. Schechter, Handbook of Analysis and Its Foundations, Academic Press, San Diego, Calif, 1997.

53. H.G. von Schnering and R. Nesper, "How Nature Adapts chemical Structures to Curved Surfaces", Angewandte Chemie 26, 1059-1200, 1987.

54. M. Schwarz, Morse Homology, Prog. in Math 111, Birkhauser Verlag, Boston, Mass, 1993.

55. V.V. Sharko, Functions on Manifolds: Algebraic and Topological Aspects, Trans. of Math. Monograms, Amer. Math. Soc., Providence, Rhode Island, 1993.

56. D. Siersma, "Voronoi Diagrams and Morse Theory of the Distance Function", Preprint 967, Department of Mathematics, University of Utrecht, 1996,

57. B. Spain, Analytical Quadrics, Pergamon, New York, NY, 1960.

58. V.Schomaker and K.N. Trueblood, Acta Cryst, B24, 63, 1968.

59. V.N. Sudakov, Geometric Problems in the Theory of Infinite-Dimensional Probability Distributions, Proc. Steklov Inst. of Math. 141, 69-177, 1976.

60. V.N. Sudakov, "Gaussian Measures", A Brief Survey, in Workshop on Measure Theory, ed. A. Volcic, Vol 26, suppl., Ediz. Elett., Rend. dell'Istit. Mate. dell'Univ. Trieste, pp. 289-325, (electronic preprint), 1994.

61. W.T. Thurston. The Geometry and Topology of Three-Manifolds, Elect. ver., Ed. S. Levy, Ch 13, 1980. http://www.msri.org/publications/books/gt3m/index.html

62. W.T. Thurston, Three-Dimensional Geometry and Topology, Vol. 1, Ed. S. Levy, Princeton Univ. Press, Princeton, NJ, 1997.

63. A.J.C. Wilson, "Arithmetic Crystal Classes", International Tables for Crystallography Volume C: Mathematical, Physical and Chemical Tables - Second Edition, Editors: E. Prince. and A.J.C. Wilson, Klewer, Boston, 1992.

64. B. Zimmermann, "Finite group actions on handlebodies and equivaliant Heegaard genus for 3-manifolds", Topology and its Appl, 43, 263-274, 1992.

65. B. Zimmermann, "Genus actions of finite groups on 3-manifolds", Mich. Math. J, 43, 593-610, 1996. 